\title{
THE COHOMOLOGY OF THE SYMMETRIC GROUPS
}

\author{
BY
}

\author{
BENJAMIN MICHAEL MANN
}

\begin{abstract}
Let $\delta_{n}$ be the symmetric group on $n$ letters and $S G$ the limit of the sets of degree +1 homotopy equivalences of the $n-1$ sphere. Let $p$ be an odd prime. The main results of this paper are the calculations of $H^{*}\left(\mathcal{S}_{n}\right.$, $Z / p)$ and $H^{*}(S G, Z / p)$ as algebras, determination of the action of the Steenrod algebra, $\mathcal{Q}(p)$, on $H^{*}\left(\mathcal{S}_{n}, Z / p\right)$ and $H^{*}(S G, Z / p)$ and integral analysis of $H^{*}\left(\mathcal{S}_{n}, Z, p\right)$ and $H^{*}(S G, Z, p)$.
\end{abstract}

0. Introduction. Let $K$ and $L$ be discrete groups with $L$ abelian. The groups $H^{n}(K, L)$ have been of interest for years. [12] and [11] first considered these cohomology groups algebraically and their relation with topological problems. The algebraic groups $H^{n}(K, L)$ are isomorphic to $H^{n}\left(B_{K}, L\right)$ where $B_{K}$ is the topological classifying space for the group $K$.

Suppose $K$ is $\delta_{n}$, the symmetric group on $n$ letters. Then $H^{*}\left(\delta_{n}, L\right)$ is especially important. In the 1950's, work on cohomology operations, [29] and [30], showed the necessity for knowledge of $H^{*}\left(\delta_{p^{\prime}}, Z / p\right)$. The construction of the $\bmod p$ Steenrod operations depends on properties of $\delta_{p}$. Furthermore the Adem relations were derived using the structure of $H^{*}\left(\delta_{p^{2}}, Z / p\right)$.

If $L$ is a ring then $H^{*}(K, L)$ is a graded ring. The homology of symmetric products, [9], [17], [20], [21], and [28], computed the groups $H^{t}\left(\mathcal{S}_{n}, Z / p\right)$ as $Z / p$ vector spaces. The graded ring structure, which was not analyzed, becomes important in later problems.

There is an interesting link that ties $\delta_{n}$ to $S G$. Recall $Q\left(S^{0}\right)=\operatorname{dir} \lim \Omega^{n} S^{n}$ is the space of "infinite loops of $S^{\infty}$ " and $S G=\operatorname{dir} \lim S G_{n}$ where $S G_{n}$ is the space of degree +1 homotopy equivalences of $S^{n-1}$. $S G$ is homotopy equivalent to the +1 component of $Q\left(S^{0}\right)$.

THEOREM. (1) There is a canonical map $\omega: B_{\delta_{\infty}}=\operatorname{dir} \lim B_{\delta_{n}} \rightarrow Q\left(S^{9}\right)_{0}$ inducing integral and $\bmod p$ homology isomorphisms.

(2) The inclusions $\delta_{n} \times \mathcal{S}_{m} \rightarrow \mathcal{S}_{n+m}$ give $H_{*}\left(\mathcal{S}_{\infty}\right)$ the structure of an algebra. $\omega_{*}$ is an algebra isomorphism and a Hopf algebra isomorphism mod $p$ where $H_{*}\left(Q\left(S^{0}\right)_{0}\right)$ is an algebra under the loop sum product.

Received by the editors October 21, 1975 and, in revised form, March 29, 1977.

AMS (MOS) subject classifications (1970). Primary 18H10; Secondary 55F40.

Key words and phrases. Cohomology of groups, classifying spaces, Steenrod algebra. 
The above theorem is contained in the work of many people including [10], [16], [22], [24], [25].

Thus $B_{\varsigma_{\infty}}$ properly interpreted is a model for $S G$.

In all that follows let $p$ be an odd prime. We will write $H^{*}(K)$ for $H^{*}(K$, $Z / p) . H^{*}(K, Z, p)$ is, by definition, [5], the $p$-primary component of $H^{*}(K$, $Z)$. In [4] the algebra structure of $H^{*}\left(\delta_{p^{2}}\right)$ is computed but the arguments do not generalize to $\delta_{p^{\prime}} ; i \geqslant 3$. The main results of this paper are the calculations of $H^{*}\left(\delta_{n}\right)$ and $H^{*}(S G)$ as algebras, determination of the action of the Steenrod algebra, $\mathcal{Q}(p)$, on $H^{*}\left(\mathcal{S}_{n}\right)$ and $H^{*}(S G)$ and integral analysis of $H^{*}\left(\delta_{n}, Z, p\right)$ and $H^{*}(S G, Z, p)$.

This paper is essentially my Stanford University Ph. D. thesis written under the direction of R. James Milgram, whom I would like to thank for his advice and encouragement. I would also like to thank the referee for his numerous helpful comments including shorter proofs for two of the propositions in §II. In addition after submission of this paper I learned that Benjamin Cooper [35] and Hùynh Mùi [36] have also studied $H^{*}\left(\delta_{p^{n}}\right)$.

I. Statement of results. It is well known that a $p$-Sylow subgroup $K_{p}$ of a finite group $K$ contains all the $p$-primary homology information; more precisely, $H^{*}(K)$ and $H^{*}(K, Z, p)$ are isomorphic to subrings of $H^{*}\left(K_{p}\right)$ and $H^{*}\left(K_{p}, Z, p\right)$ respectively, which are invariant under the action of certain automorphisms. It is also well known, [6], that a $p$-Sylow subgroup of $\delta_{p^{\prime}}$ is isomorphic to $\mathrm{wr}^{i} Z / p$, the $i$-fold wreath product of $Z / p$. In the next section we examine a specific embedding of $w^{i} Z / p$ in $\delta_{p^{\prime}}$ and show the existence of an $H^{*}()$ detecting family consisting of subgroups of the form $\times^{m} Z / p$. In fact we have the following subgroups and natural inclusions: $k_{j, i}: T_{j, i} \rightarrow \delta_{p^{\prime}}$ for $1 \leqslant j \leqslant i$ and the map $k_{i}^{*}=\prod_{j=1}^{i} k_{j, i}^{*}: H^{*}\left(\delta_{p^{\prime}}\right) \rightarrow \prod_{j=1}^{i} H^{*}\left(T_{j, i}\right)$, where $T_{j, i}=\times^{p^{i-j}}\left(\times^{j} Z / p\right)$.

The first theorems compute the images of $k_{j, i}^{*}$ 's and the map $k_{i}^{*}$. We show that $k_{i}^{*}$ detects a set of multiplicative generators for $H^{*}\left(\delta_{p^{i}}\right)$ whose relations are trivial to compute. Hence the map $k_{i}^{*}$ determines $H^{*}\left(\delta_{p^{i}}\right)$. Later for simplicity we will want to identify $u \in H^{*}\left(\delta_{p^{\prime}}\right)$ with its natural image $k_{j, i}^{*}(u) \in H^{*}\left(T_{j, i}\right)$ but we must wait until Theorems A-D have been stated to avoid possible confusion.

Recall $H^{*}\left(X^{k} Z / p\right)=E\left(e_{1}, \ldots, e_{k}\right) \otimes P\left(b_{1}, \ldots, b_{k}\right)$ with degree $\left(e_{m}\right)=$ 1 , degree $\left(b_{m}\right)=2$ for all $m$. Furthermore $\beta_{p}\left(e_{m}\right)=b_{m}$, where $\beta_{p}$ is the Bockstein operator associated with the exact coefficient sequence $0 \rightarrow Z / p \rightarrow$ $Z / p^{2} \rightarrow Z / p \rightarrow 0$.

Consider the following classes in $H^{*}\left(\times^{i} Z / p\right)$ : (a matrix cohomology class will always mean the cohomology class given by the formal determinant of that matrix) 


$$
\begin{aligned}
& L_{i}=\left|\begin{array}{ccc}
b_{1}^{p^{-1}} & \cdots & b_{i}^{p^{-1}} \\
\vdots & \vdots & \vdots \\
b_{1}^{p^{\prime}} & \cdots & b_{i}^{p^{r}} \\
\vdots & \vdots & \vdots \\
b_{1} & \cdots & b_{i}
\end{array}\right| \quad \text { is. the } k, j \text { entry of } L_{i} \\
& Q_{j, i}=\frac{\left|\begin{array}{ccc}
b_{1}^{p^{\prime}} & \cdots & b_{i}^{p^{\prime}} \\
\vdots & \vdots & \vdots \\
\widehat{b_{1}^{p}} & \cdots & \vec{b}_{i}^{p^{\prime}} \\
\vdots & \vdots & \vdots \\
b_{1} & \cdots & b_{i}
\end{array}\right| \begin{array}{l}
\text { i.e. the } b^{p^{\prime}} \text { row of the } \\
(1<j \leqslant i-1) .
\end{array}}{L_{i}} \\
& \underline{L}_{i}=\left|\begin{array}{ccc}
b_{1}^{p^{\prime-1}} & \cdots & b_{1}^{p^{\prime-1}} \\
\vdots & & \vdots \\
b_{1}^{p} & \cdots & b_{i}^{p} \\
e_{1} & \cdots & e_{i}
\end{array}\right| \begin{array}{l}
\text { i.e. } \underline{L}_{i} \text { is the } L_{i} \text { determinant } \\
\text { with the } b_{1} \cdots b_{i} \text { row replaced } e_{1} \cdots e_{i} .
\end{array}
\end{aligned}
$$

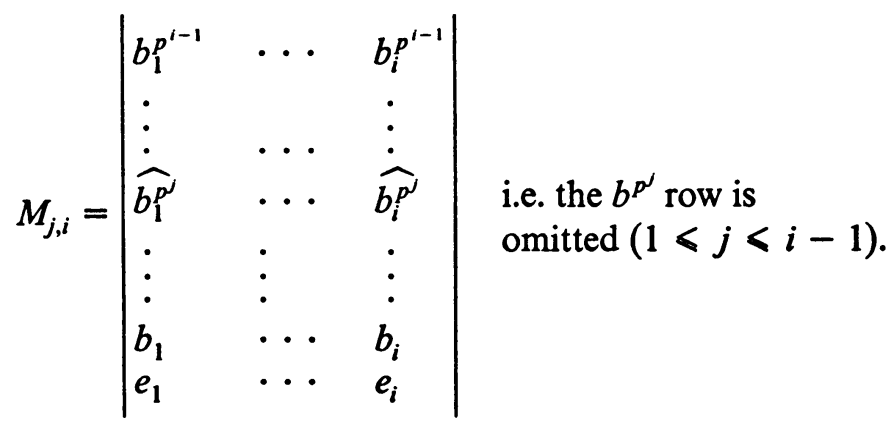

Note. (i) If $i=1$ then $L_{1}=b_{1}$ and $\underline{L}_{1}=e_{1}$ are the only two classes defined. (ii) [19] proved $Q_{j, i}$ is integral, not merely rational, mod $p$. See appendix for proof.

$\delta_{p^{\prime}}$ can be thought of as the permutations of the point set $\Pi^{i} Z / p$. Let $k_{i, i}$ : $T_{i, i}=X^{i} Z / p \rightarrow$ permutations of $\left.\Pi^{i} Z / p\right\}$ be defined by: $k_{i, i}\left(a_{1}, \ldots, a_{i}\right)$ sends $\left(b_{1}, \ldots, b_{i}\right)$ to $\left(a_{1}+b_{1}, \ldots, a_{i}+b_{i}\right)$ where $Z / p$ is written additively. Then $k_{i, i}$ is seen to be equivalent to the adjoint representation (2.5) and 
includes $T_{i, i}$ in $\delta_{p^{i}}$. The normalizer $N$ of $k_{i, i}\left(T_{i, i}\right)$ in $\delta_{p^{\prime}}$ maps onto GL $(i, Z / p)$ (2.10) and induces an action on $H^{*}\left(T_{i, i}\right)$ as follows. If $\cup_{x}$ in $\mathrm{GL}(i, Z / p)$ represents the coset $x T_{i, i}$ in $N$ then the homomorphism $\operatorname{ad}_{x}: H^{*}\left(T_{i, i}\right) \rightarrow$ $H^{*}\left(T_{i, i}\right)$ operates as follows: $\operatorname{ad}_{x}\left(e_{m}\right)=\cup_{x} e_{m}, a_{x}\left(b_{m}\right)=\cup_{x} b_{m}$ where $e_{m}$, $b_{m}$ are treated as the vectors $(0, \ldots, e, \ldots, 0)$ and $(0, \ldots, b, \ldots, 0)$ in $H^{*}\left(T_{i, i}\right)$ with nonzero entries in the $m$ th place. Hence $\mathrm{ad}_{x}$ operates on the above determinant classes via the determinant function; that is, $\operatorname{ad}_{x}\left(L_{i}\right)=$ $\operatorname{det}\left(\cup_{x} L_{i}\right)$. By 2.13 image $k_{i, i}^{*}$ is contained in $H^{*}\left(T_{i, i}\right)^{\mathrm{GL}(i, Z / p)}$.

Let $w_{1}$ be the algebra $E\left(\underline{L}_{1} L_{1}^{p-2}\right) \otimes P\left(L_{1}^{p-1}\right)$. For $i$ greater than 1 let $\mho_{i}$ be the subalgebra of $H^{*}\left(T_{i, i}\right)$ generated by: $1, L_{i}^{p-1}, Q_{j, i}, L_{i} L_{i}^{p-2}, M_{j, i} L_{i}^{p-2}$, $M_{j, i} \underline{L}_{i} L_{i}^{p-3}, M_{j, i} M_{h, i} L_{i}^{p-3}$ with $1 \leqslant j, h \leqslant i-1$ and $j<h$. ow $S_{i}$ is contained in $H^{*}\left(T_{i, i}\right)^{\mathrm{GL}(i, Z / p)}(2.12)$. Then $\mathscr{W}_{i}$ contains the polynomial algebra $P\left(L_{i}^{p-1}, Q_{1, i}, Q_{2, i}, \ldots, Q_{i-1, i}\right)$ and all other generators of $\mathscr{W}_{i}$ are exterior. However the algebra they generate is not an exterior subalgebra as there are zero products. The multiplication of these exterior products is determined by the relations:

(1) $\underline{L}_{i}^{2}=M_{j, i}^{2}=0,1 \leqslant j \leqslant i-1$,

(2) $\underline{L}_{i} M_{1, i} M_{2, i} \cdots M_{i-1, i} \neq 0$.

For example $\left(M_{2, i} M_{3, i} L_{i}^{p-3}\right)\left(M_{2, i} M_{5, i} L_{i}^{p-3}\right)=0$.

THEOREM A. image $k_{i, i}^{*} \cong W_{i}$.

EXAMPLES. (i) If $i=1$ then $0 \rightarrow H^{*}\left(\mathcal{S}_{p}\right) \rightarrow^{k_{i, 1}^{*}, H^{*}}(Z / p)$ where $H^{*}(Z / p) \cong$ $E\left(\underline{L}_{1}\right) \otimes P\left(L_{1}\right)$ and $H^{*}\left(\delta_{p}\right) \cong E\left(\underline{L}_{1} L_{1}^{p-2}\right) \otimes P\left(L_{1}^{p-1}\right)$.

(ii) If $i=2$ the results of [4] are obtained.

(iii) Let $p=3, i=3$ then $k_{3,3}^{*}: H^{*}\left(\mathcal{S}_{27}\right) \rightarrow H^{*}(Z / 3 \times Z / 3 \times Z / 3)$ and image $k_{3,3}^{*}$ is generated by:

(1) polynomial generators

$$
\begin{gathered}
L_{3}^{2}=\left|\begin{array}{lll}
b_{1}^{9} & b_{2}^{9} & b_{3}^{9} \\
b_{1}^{3} & b_{2}^{3} & b_{3}^{3} \\
b_{1} & b_{2} & b_{3}
\end{array}\right|^{2} \quad Q_{1,3}=\frac{\left|\begin{array}{lll}
b_{1}^{27} & b_{2}^{27} & b_{3}^{27} \\
b_{1}^{9} & b_{2}^{9} & b_{3}^{9} \\
b_{1} & b_{2} & b_{3}
\end{array}\right|}{L_{3}}, \\
Q_{2,3}=\frac{\left|\begin{array}{lll}
b_{1}^{27} & b_{2}^{27} & b_{3}^{27} \\
b_{1}^{3} & b_{2}^{3} & b_{3}^{3} \\
b_{1} & b_{2} & b_{3}
\end{array}\right|}{L_{3}} .
\end{gathered}
$$

(2) exterior generators 


$$
M_{1,3} M_{2,3}=\left|\begin{array}{lll}
b_{1}^{9} & b_{2}^{9} & b_{3}^{9} \\
b_{1} & b_{2} & b_{3} \\
e_{1} & e_{2} & e_{3}
\end{array}\right|\left|\begin{array}{lll}
b_{1}^{3} & b_{2}^{3} & b_{3}^{3} \\
b_{1} & b_{2} & b_{3} \\
e_{1} & e_{2} & e_{3}
\end{array}\right|,
$$

$M_{1,3} \underline{L}_{3}, M_{1,3} L_{3}, M_{2,3} \underline{L}_{3}, M_{2,3} L_{3}, \underline{L}_{3} L_{3}$.

(3) the relations that any product of exterior generators is zero except

(a) $\left(M_{1,3} M_{2,3}\right)\left(\underline{L}_{3} L_{3}\right)=-\left(M_{1,3} \underline{L}_{3}\right)\left(M_{2,3} L_{3}\right)=\left(M_{2,3} \underline{L}_{3}\right)\left(M_{1,3} L_{3}\right)$,

(b) $\left(M_{1,3} L_{3}\right)\left(\underline{L}_{3} L_{3}\right)=\left(M_{1,3} \underline{L}_{3}\right) L_{3}^{2}$,

(c) $\left(M_{2,3} L_{3}\right)\left(\underline{L}_{3} L_{3}\right)=\left(M_{2,3} \underline{L}_{3}\right) L_{3}^{2}$,

(d) $\left(M_{1,3} L_{3}\right)\left(M_{2,3} L_{3}\right)=\left(M_{1,3} M_{2,3}\right) L_{3}^{2}$.

The proof of Theorem A depends, in part, on [17] and a counting argument. As noted above the classes in image $k_{i, i}^{*}$ are $\mathrm{GL}(i, Z / p)$ invariant. A calculation and [8] show $P\left(b_{1}, \ldots, b_{i}\right)^{\mathrm{GL}(i, Z / p)}$ is isomorphic to the polynomial subalgebra of image $k_{i, i}^{*}$. For $i=2$, [4] shows

$$
\left(E\left(e_{1}, e_{2}\right) \otimes P\left(b_{1}, b_{2}\right)\right)^{\mathrm{GL}(2, Z / p)} \cong H^{*}(Z / p \times Z / p)^{\mathrm{GL}(2, Z / p)} \cong \text { image } k_{2,2}^{*} \text {. }
$$

If $p \geqslant 5, i \geqslant 3$ then $\left(E\left(e_{1}, \ldots, e_{i}\right) \otimes P\left(b_{1}, \ldots, b_{i}\right)\right)^{\mathrm{GL}(i, Z / p)}$ properly contains image $k_{i, i}^{*}$; for example, $M_{1, i} M_{2, i} L_{i} L_{i}^{p-4}$ is not in image $k_{i, i}^{*}$. For $p=3$, $i \geqslant 3$, it is unknown if image $k_{i, i}^{*}$ equals the ring of invariants.

Consider the inclusion $X_{m=1}^{p}\left(\mathcal{S}_{p^{i-1}}\right)_{m} \rightarrow^{I_{i-1}} \mathcal{S}_{p^{\prime}}$ where $\left(\mathscr{S}_{p^{i-1}}\right)_{m}$ permutes the $p^{i-1}$ letters $\left((m-1) p^{i-1}+1, \ldots, m p^{i-1}\right)$. Then let $k_{i-1, i}: T_{i-1, i} \rightarrow \delta_{p^{i}}$ be the composition $I_{i-1}\left(X_{m=1}^{p}\left(k_{i-1, i-1}\right)_{m}\right)$. More generally let $k_{j, i}: T_{j, i} \rightarrow \mathcal{S}_{p^{\prime}}$ be the composition $I_{j}\left(X_{m=1}^{p^{i-j}}\left(k_{j, j}\right)_{m}\right)$ where $I_{j}$ is the inclusion $X_{m=1}^{p^{i-j}}\left(\delta_{p^{j}}\right)_{m} \rightarrow \delta_{p^{\prime}}$ given by letting $\left(\delta_{p^{j}}\right)_{m}$ permute the $m$ th block of $p^{j}$ letters.

Let $1 \leqslant j \leqslant i$, then $\delta_{p^{\prime-j}}$ operates on $T_{j, i}$ and on the algebra $\otimes_{m=1}^{p^{\prime-j}}\left(\vartheta_{j}\right)_{m}$ contained in $H^{*}\left(T_{j, i}\right) \cong \bigotimes \underset{m=1}{p_{m=1}^{i-j}}\left(H^{*}\left(X^{j} Z / p\right)\right)_{m}$ by permuting the $p^{i-j}$ copies of $\times^{j} Z / p$.

THEOREM B. For $1 \leqslant j \leqslant i$ image $k_{j, i}^{*}$ is isomorphic to the algebra of $\widehat{S}_{p^{i-j}}$ invariant classes of $\otimes_{m=1}^{p^{i-j}}\left(\mathcal{W}_{j}\right)_{m}$.

Notation. Let $u_{m} \in\left(\mho_{j}\right)_{m}$ then $\delta\left\langle u_{1}, u_{2}, \ldots, u_{p^{i-j}}\right\rangle$ is the $\delta_{p^{i-j}}$ invariant class generated by $u_{1} u_{2} \cdots u_{p^{\prime-\jmath}}\left(u_{m}\right.$ is allowed to be $\left.1 \in H^{0}\left(X^{j} Z / p\right)\right)$. If $u_{1}$ is odd dimensional then $\mathcal{S}\left\langle u_{1}, u_{1}, \ldots, u_{p^{\prime-j}}\right\rangle=0$.

EXAMPLES. (i) image $k_{1,1}^{*}$ is generated by:

$$
\begin{aligned}
A_{k, i} & =\sum_{m=1}^{p^{i-1}}\left(\underline{L}_{1} L_{1}^{(p-2)+k(p-1)}\right)_{m} \\
& =\delta\left\langle\left(\underline{L}_{1} L_{l}^{(p-2)+k(p-1)}\right), 1, \ldots, 1\right\rangle, \text { for } 0 \leqslant k \leqslant p^{i-1}-1,
\end{aligned}
$$

and

$$
B_{k, i}=\sum\left(L_{1}^{p-1}\right)_{m_{1}}\left(L_{1}^{p-1}\right)_{m_{2}} \cdots\left(L_{1}^{p-1}\right)_{m_{k}},
$$

where $1 \leqslant k \leqslant p^{i-1}$ and the sum runs over all sequences $1 \leqslant m_{1}<m_{2}$ 
$<\cdots<m_{k} \leqslant p^{i-1}$. Thus $B_{k, i}=\delta\left\langle L_{1}^{p-1}, L_{1}^{p-1}, \ldots, L_{1}^{p-1}, 1, \ldots, 1\right\rangle$ where $L_{1}^{p-1}$ appears $k$ times.

(ii) Let $p=3$, then $k_{2,3}^{*}: H^{*}\left(\mathcal{S}_{27}\right) \rightarrow H^{*}\left(T_{2,3}\right)$ and image $k_{2,3}^{*}$ is generated by:
$\delta\langle$ ext, 1,1$\rangle$
$\delta\langle$ poly, 1, 1〉
$\delta\langle$ ext, poly, 1$\rangle$
$\delta\left\langle M_{1,2} \underline{L}_{2}, M_{1,2} \underline{L}_{2}, M_{1,2} \underline{L}_{2}\right\rangle$
$\delta\langle$ ext, poly, poly $\rangle$
$\delta\langle$ poly, poly, 1$\rangle$
$\delta\langle$ poly, poly, poly〉
$\delta\langle$ ext, ext, poly $\rangle$

where

(a) ext runs through $M_{1,2} \underline{L}_{2}, M_{1,2} L_{2}$, and $\underline{L}_{2} L_{2}$.

(b) poly runs through $L_{2}^{2}$ and $Q_{1,2}$.

(c) As $M_{1,2} L_{2}$ and $\underline{L}_{2} L_{2}$ are odd dimensional neither can appear twice in any $\delta\langle-,-,-\rangle$. For example $\delta\left\langle\underline{L}_{2} L_{2}, \underline{L}_{2} L_{2}, 1\right\rangle=0$. Note that $\delta\left\langle M_{1,2} \underline{L}_{2}\right.$, $1,1\rangle$ has height 3 while $\delta\left\langle M_{1.2} L_{2}, 1,1\right\rangle$ is exterior.

(iii) In image $k_{2, i}^{*}$ the classes

$$
\delta\left\langle M_{1,2} \underline{L}_{2} L_{2}^{p-3}, 1, \ldots, 1\right\rangle
$$

and

$$
\delta\left\langle\left(M_{1,2} \underline{L}_{2} L_{2}^{p-3}\right)_{1}, \ldots,\left(M_{1,2} \underline{L}_{2} L_{2}^{p-3}\right)_{p}, 1, \ldots, 1\right\rangle
$$

have height $p$ while $\delta\left\langle M_{1,2} \underline{L}_{2} L_{2}^{p-3}, \ldots, M_{1,2} \underline{L}_{2} L_{2}^{p-3}\right\rangle$ is exterior. This pattern generalizes to image $k_{j, i}^{*}, 3 \leqslant j \leqslant i-1$, in the obvious way.

Note. Example (iii) shows how all even dimension exterior generators in $w_{j}$ build classes in $H^{*}\left(T_{j, i}\right)$ which are the images under $k_{j, i}^{*}$ of classes $u \in$ $H^{*}\left(\delta_{p^{\prime}}\right)$ where each $u$ generates a truncated polynomial algebra of height $p$ in $H^{*}\left(\mathcal{S}_{n}\right)$. These are the truncated polynomial algebras described in [22].

Let $u \in H^{*}\left(\delta_{p^{i}}\right)$ then $k_{i}^{*}(u)=\left(k_{1, i}^{*}(u), \ldots, k_{i, i}^{*}(u)\right)$ and the algebra structure restricted to these detecting groups is compatible with component-wise projection. Clearly to calculate $H^{*}\left(\mathscr{S}_{p^{i}}\right)$ we must know when a class $u \in$ $H^{*}\left(\delta_{p^{\prime}}\right)$ has nontrivial image under more than one $k_{i, j}^{*}$.

Definition. $u \in H^{*}\left(\delta_{p^{\prime}}\right)$ is a multiple image class if and only if $k_{j, i}^{*}(u) \neq 0$ for at least two different values of $j$.

Given $u_{1}, u_{2} \in H^{*}\left(\delta_{p^{i}}\right)$ with $u_{1}$ detected only by $k_{j_{1}, i}^{*}$ and $u_{2}$ detected only by $k_{j_{2}, i}^{*}$ with $j_{1} \neq j_{2}$ then $u_{1}+u_{2}$ is a multiple image class. However this type of multiple image class is decomposable as a sum of classes and thus is a "trivial" multiple image class. The next three definitions and following theorem give all "nontrivial"; i.e., indecomposable, multiple image classes.

DEFINITION. $\mathfrak{T}_{i}$ is the subalgebra contained in $\mathcal{W}_{i}$ generated by 1 , $M_{g, i} M_{h, i} L_{i}^{p-3}, Q_{h, i}, 1 \leqslant g, h \leqslant i-1, g<h$.

DefinITION. Given $x_{m_{j}} \in \mathfrak{N}_{j}$ we define $x_{m_{j-1}} \in \mathcal{W}_{j-1}$ as follows:

(a) If $x_{m, j}=1$ then $x_{m, j-1}=1$. 
(b) If $x_{m, j}=Q_{h, j}$ then $x_{m, j-1}=Q_{h-1, j-1}$, for $2 \leqslant j \leqslant i$ and $1 \leqslant h \leqslant j-1$ with the convention $Q_{0, j-1}=L_{j-1}^{p-1}$.

(c) If $x_{m, j}=M_{g, j} M_{h, j} L_{j}^{p-3}$ then $x_{m, j-1}=-M_{g-1, j-1} M_{h-1, j-1} L_{j-1}^{p-3}$, for $3 \leqslant$ $j \leqslant i, 0<g, h<j$ and $g<h$ with the convention $M_{0, j-1}=\underline{L}_{j-1}$.

(d) If $x_{m, j}=x_{m, j}^{\prime} x_{m, j}^{\prime \prime}$ then $x_{m-1, j-1}=x_{m-1, j-1}^{\prime} x_{m-1, j-1}^{\prime \prime}$.

Note. (a) through (d) define a unique class $x_{m, j-1}$ for every $x_{m, j} \in \mathfrak{N}_{j}$.

DefinItION. $u \in H^{*}\left(\mathscr{S}_{n}\right)$ is sum indecomposable if and only if $u=u_{1}+u_{2}$ for $u_{1}, u_{2} \in H^{*}\left(\mathscr{S}_{n}\right)$ implies $u_{1}$ or $u_{2}$ is zero.

THEOREM C. Suppose $u \in H^{*}\left(\delta_{p^{\prime}}\right)$ is both sum indecomposable and a multiple image class. Further suppose $j$ is the largest integer such that $k_{j, i}^{*}(u) \neq 0$. Then

$$
k_{j, i}^{*}(u)=\mathcal{S}\left\langle x_{1, j}, \ldots, x_{p^{i-j}, j}\right\rangle
$$

with $x_{m, j} \in \mathfrak{N}_{j}$ for $1 \leqslant m \leqslant p^{1-j}$, and

$$
k_{j-1, i}^{*}(u)=\delta\left\langle x_{1, j-1}, \ldots, x_{1, j-1}, \ldots, x_{p^{i-j}, j-1}, \ldots, x_{p^{i-j}, j-1}\right\rangle
$$

where each $x_{m j-1}$ is as defined above and appears $p$ times in $k_{j-1, i}^{*}(u)$. If $j-1 \geqslant 2$ and each $x_{m, j-1} \in \mathfrak{N}_{j-1}$ (not just $\mathcal{W}_{j-1}$ ) then $k_{j-2, i}^{*}(u) \neq 0$ and may be obtained from $k_{j-1, i}^{*}(u)$ precisely as $k_{j-1, i}^{*}(u)$ was obtained from $k_{j, i}^{*}(u)$. In fact this iteration continues $r$ times until either $j-r=2$ or $x_{m j-r} \notin \mathfrak{N}_{j-r}$ when $k_{j-(r+t), i}^{*}(u)=0$ for all $t>0$. Thus $u$ has $r+1$ nontrivial images in the detecting groups: $k_{j-s, i}^{*}$ for $0 \leqslant s \leqslant r$.

EXAMPLE. For $H^{*}\left(S_{27}, Z / 3\right)$ the only sum-indecomposable multiple image classes of $k_{0}^{*}$ occurring as generators in the examples after Theorems $\mathrm{A}$ and $\mathrm{B}$ are:

$\left(B_{9},\left(Q_{1,2}\right)_{1}\left(Q_{1,2}\right)_{2}\left(Q_{1,2}\right)_{3}, Q_{2,3}\right)$

$\left(0,\left(L_{2}^{2}\right)_{1}\left(L_{2}^{2}\right)_{2}\left(L_{2}^{2}\right)_{3}, Q_{1,3}\right)$,

$\left(0,\left(M_{1,2} \underline{L}_{2}\right)_{1}\left(M_{1,2} \underline{L}_{2}\right)_{2}\left(M_{1,2} \underline{L}_{2}\right)_{3},-M_{1,3} M_{2,3}\right)$,

$\left(B_{3}, \delta\left\langle Q_{1,2}, 1,1\right\rangle, 0\right)$,

$\left(B_{6}, \delta\left\langle Q_{1,2}, Q_{1,2}, 1\right\rangle, 0\right)$.

Consider $u_{1} u_{2}$ in $H^{*}\left(\delta_{p^{3}}\right)$ where $k_{3}^{*}\left(u_{1}\right)=\left(\delta\left\langle L_{1}^{p-1}, 1, \ldots, 1\right\rangle, 0,0\right)$ and $k_{3}^{*}\left(u_{2}\right)=\left(0, \delta\left\langle L_{2}^{p-1}, 1, \ldots, 1\right\rangle, 0\right)$. Then $k_{3}^{*}\left(u_{1} u_{2}\right)=0$ but in fact $u_{1} u_{2} \neq 0$ in $H^{*}\left(\delta_{p^{3}}\right)$ and $u_{1} u_{2}$ is detected by subgroups of the form $T_{1} \times T_{2} \times \cdot \cdot \times$ $T_{p}$ where $T_{n}=T_{1,2}$ or $T_{2,2}$ and both $T_{1,2}$ and $T_{2,2}$ must occur at least once. These detecting groups are included in $\delta_{p^{3}}$ through $X^{p}\left(\delta_{p^{2}}\right)$. More generally a nonsymmetric detecting group, $X_{n=1}^{p}\left(\times_{m=1}^{t}\left(T_{r_{m}, s_{m}}\right)\right)_{n}$ of $\delta_{p^{\prime}}$ is a product of detecting groups of $\delta_{p^{i-1}}$ included in $\delta_{p^{\prime}}$ through $\times^{p}\left(\delta_{p^{i-1}}\right)$ where $T_{r_{1}, S_{1}} \neq$ $T_{r_{2}, s_{2}}$ for some $r_{1}, r_{2}, s_{1}$ and $s_{2}$. These nonsymmetric detecting groups detect all classes $u \in H^{*}\left(\mathcal{S}_{p^{i}}\right)$ not detected by the map $k_{i}^{*}$ as stated in Theorem D. First we need 
Definition. Let $u \in H^{*}\left(\mathcal{S}_{p^{i}}\right)$ and $n<p^{i}$. Then we have the natural inclusion $I_{p^{i}, n}: \delta_{n} \hookrightarrow \delta_{p^{i}}$. We say $u$ restricts nonzero to $\delta_{n}$ if and only if $I_{p^{i}, n}^{*}(u) \neq 0$. For notational convenience we write $u$ for both the class in $H^{*}\left(\delta_{p^{\prime}}\right)$ and the restriction in $H^{*}\left(\mathcal{S}_{n}\right)$.

THEOREM D. (1) The classes in $H^{*}\left(\delta_{p^{\prime}}\right)$ not detected by $k_{i}^{*}$ are products of classes that are detected by $k_{i}^{*}$.

(2) Let $u_{m} \in H^{*}\left(\delta_{p^{i}}\right)$. Suppose $k_{i}^{*}\left(u_{m}\right) \neq 0, \Pi_{m=1}^{r} k_{i}^{*}\left(u_{m}\right)=0$ and let $n_{m}$ be the smallest power of $p$ such that $u_{m}$ restricts nonzero to $H^{*}\left(\delta_{n_{m}}\right)$. Then $\Pi_{m=1}^{r} u_{m} \neq 0$ in $H^{*}\left(\delta_{p^{i}}\right)$ unless:

(a) $u_{m_{1}}=u_{m_{2}}$ is an odd dimensional exterior class in $H^{*}\left(\delta_{n_{m_{1}}}\right)$, for some $1 \leqslant m_{1}<m_{2} \leqslant r$.

(b) $u_{m_{1}}=u_{m_{2}}=\cdots=u_{m_{p}}$ is an even dimensional exterior class in $H^{*}\left(\mathcal{S}_{n_{m_{1}}}\right)$ for some $1 \leqslant m_{1}<m_{2}<\cdots<m_{p} \leqslant r$ or

(c) $\delta_{n_{1}} \times \cdots \times \delta_{n_{r}}$ is not contained in $\delta_{p^{\prime}}$.

Note. The classes $u_{m_{1}}$ appearing in condition (b) are the generators for the truncated polynomial algebras described in example (iii) after Theorem B.

Thus every $u \in H^{*}\left(\delta_{p^{\prime}}\right)$ is expressible as a sum of monomials $\sum a\left(u_{1}, \ldots, u_{r}\right) u_{1} \otimes \cdots \otimes u_{r}$ where $a\left(u_{1}, \ldots, u_{r}\right) \in Z / p, u_{t} \in H^{*}\left(\delta_{p^{i}}\right)$ with $k_{i}\left(u_{t}\right) \neq 0$ for all $t$.

Definition. $u \in H^{*}\left(\mathcal{S}_{p^{i}}\right)$ is proper if and only if $u=\Sigma a\left(u_{1}, \ldots, u_{r}\right) u_{1}$ $\otimes \cdots \otimes u_{r}$ with $k_{i}^{*}\left(u_{1} \otimes \cdots \otimes u_{r}\right) \neq 0$ for each monomial in the sum.

Thus Theorems A through D compute $H^{*}\left(\delta_{p^{i}}\right)$ and from this point on we will identify elements of $H^{*}\left(\delta_{p^{\prime}}\right)$ with their image under $k_{i}^{*}$. That is $L_{i}^{p-1} Q_{j, i}$ $\in H^{*}\left(\delta_{p^{\prime}}\right)$ is the unique proper class $u \in H^{*}\left(\delta_{p^{\prime}}\right)$ such that $k_{i}^{*}(u)=$ $\left(0, \ldots, 0, L_{i}^{p-1} Q_{j, i}\right)$. Care must be taken with multiple image classes under this identification. Notice, by Theorem $\mathrm{C}$, that $Q_{1, i} \in H^{*}\left(\delta_{p^{\prime}}\right)$ is the unique proper class $u \in H^{*}\left(\delta_{p^{i}}\right)$ such that $k_{i}^{*}(u)=\left(0, \ldots, 0, \delta\left\langle L_{i-1}^{p-1}, \ldots, L_{i-1}^{p-1}\right\rangle\right.$, $\left.Q_{1, i}\right)$.

Since

$$
\mathscr{P}^{j}\left(b^{p^{k}}\right)= \begin{cases}b^{p^{k}} & \text { if } j=0 \\ b^{p^{k+1}} & \text { if } j=p^{k} \\ 0 & \text { otherwise }\end{cases}
$$

it is easy to determine the action of the Steenrod algebra $\mathbb{Q}(p)$ on $H^{*}\left(\mathcal{S}_{p^{i}}\right)$. Consider $M_{1,3} L_{3}$ in $H^{47}\left(\delta_{27}, Z / 3\right)$. Then

$$
\mathscr{P}^{1}\left(\left|\begin{array}{lll}
b_{1}^{9} & b_{2}^{9} & b_{3}^{9} \\
b_{1} & b_{2} & b_{3} \\
e_{1} & e_{2} & e_{3}
\end{array}\right|\left|\begin{array}{ccc}
b_{1}^{9} & b_{2}^{9} & b_{3}^{9} \\
b_{1}^{3} & b_{2}^{3} & b_{3}^{3} \\
b_{1} & b_{2} & b_{3}
\end{array}\right|\right)=\left|\begin{array}{lll}
b_{1}^{9} & b_{2}^{9} & b_{3}^{9} \\
b_{1}^{3} & b_{2}^{3} & b_{3}^{3} \\
e_{1} & e_{2} & e_{3}
\end{array}\right|\left|\begin{array}{ccc}
b_{1}^{9} & b_{2}^{9} & b_{3}^{9} \\
b_{1}^{3} & b_{2}^{3} & b_{3}^{3} \\
b_{1} & b_{2} & b_{3}
\end{array}\right|=\underline{L}_{3} L_{3} .
$$


This computation involved use of the Cartan formula; however, all terms except the first are zero. The next theorem describes the $\mathcal{Q}(p)$ action on $\mathscr{W}_{i}$. Note the polynomial subalgebra of $\mathscr{W}_{i}$ is closed under the $\mathscr{Q}(p)$ action while a class in the ideal generated by the exterior generators of $\mho_{i}$ may be "bocksteined" into the polynomial algebra; e.g., $\beta \mathscr{P}^{1}\left(M_{1, i} L_{i}^{p-2}\right)=L_{1}^{p-1}$ for $i>1$. Using the Cartan formula and the following theorem it is trivial to compute the $\mathcal{Q}(p)$ action on all the detecting groups.

THEOREM E. The following relations and the Cartan formula describe the $\mathcal{Q}(p)$ action on $\mathcal{W}_{i}$.

(1) $\mathscr{P}^{p^{h-1}}\left(M_{j, i} M_{h, i} L_{i}^{p-3}\right)=M_{j, i} M_{h-1, i} L_{i}^{p-3}, j>h$ and $M_{0, i}=\underline{L}_{i}$,

(2) $\odot P^{p^{j-1}}\left(M_{j, i} M_{h, i} L_{i}^{p-3}\right)=M_{j-1, i} M_{h, i} L_{i}^{p-3}, j>h$ and $M_{0, i}=\underline{L}_{i}$,

(3) $\beta\left(\underline{L}_{i}\right)=L_{i}$,

(4) $\operatorname{SPp}^{h-1}\left(Q_{h, i}\right)=Q_{h-1, i}$, with $Q_{0, i}=L_{i}^{p-1}$,

(5) $\mathcal{P}^{p^{i-1}}\left(L_{i}^{p-1}\right)=-Q_{i-1, i} L_{i}^{p-1}$ for $i>1$ while

$\mathscr{P}^{j}\left(L_{1}^{p-1}\right)=\left(\begin{array}{c}p-1 \\ j\end{array}\right) L_{1}^{(p-1)(j+1)}$ for $j \leqslant p-1$.

(6) $\rho^{p^{i-1}}\left(M_{i-1, i} L_{i} L_{i}^{p-3}\right)=(p-2)\left(M_{i-1, i} L_{i} L_{i}^{p-3}\right)\left(Q_{i-1, i}\right)$

$\mathscr{P}^{p^{i-1}}\left(M_{i-1, i} L_{i}^{p-2}\right)=(p-2)\left(M_{i-1, i} L_{i}^{p-2}\right)\left(Q_{i-1, i}\right)$.

The following diagram is conceptually helpful.

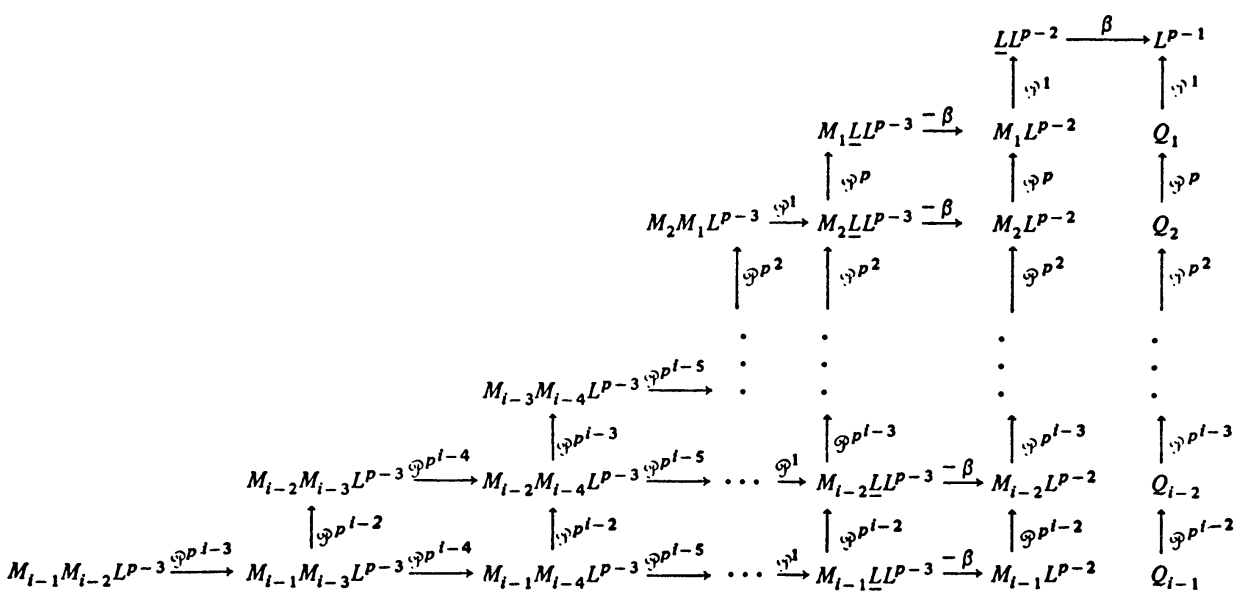

THE ACTION OF $\mathcal{Q}(p)$ ON THE GENERATORS OF $w_{i}$

EXAMPLES. (i) Consider $A=\left(0, \delta\left\langle M_{1,2} \underline{L}_{2}, M_{1,2} \underline{L}_{2}, M_{1,2} \underline{L}_{2}\right\rangle,-M_{2,3} M_{1,3}\right)$ in $H^{30}\left(\mathcal{S}_{27}, Z / 3\right)$. Then

$$
\mathcal{P}^{1} \beta(A)=\left(0,-\delta\left\langle\underline{L}_{2} L_{2}, M_{1,2} \underline{L}_{2}, M_{1,2} \underline{L}_{2}\right\rangle, 0\right)
$$

while

$$
\beta^{\mathcal{P}}(A)=\left(0,0, M_{2,3} L_{3}\right) .
$$


(ii)

$$
\begin{aligned}
\mathcal{P}^{p^{i-2}} \mathcal{P p}^{p^{-1}}\left(M_{i-1, i} L_{i}^{p-2}\right) & =\mathcal{P}^{p^{i-2}}\left((p-2)\left(M_{i-1, i} L_{i}^{p-2}\right) Q_{i-1, i}\right) \\
= & (p-2)\left[\left(M_{i-2, i} L_{i}^{p-2}\right) Q_{i-1, i}+\left(M_{i-1, i} L_{i}^{p-2}\right) Q_{i-2, i}\right] .
\end{aligned}
$$

Let $n$ be an arbitrary integer. Then $n$ may be written uniquely as follows: $n=\sum_{j=0}^{i} a_{j} p^{j}$ with $0 \leqslant a_{j} \leqslant p-1, a_{i} \neq 0$. A $p$-Sylow subgroup $K_{p}$ of $S_{n}$ is isomorphic to

$$
K_{p}=\stackrel{a_{i}}{\times}(\stackrel{i}{\mathrm{wr}} Z / p) \times \stackrel{a_{i-1}}{\times}(\stackrel{i-1}{\mathrm{wr}} Z / p) \times \cdots \times \stackrel{a_{1}}{\times}(Z / p) .
$$

To compute $H^{*}\left(\Im_{n}\right)$ consider the following diagram of inclusions

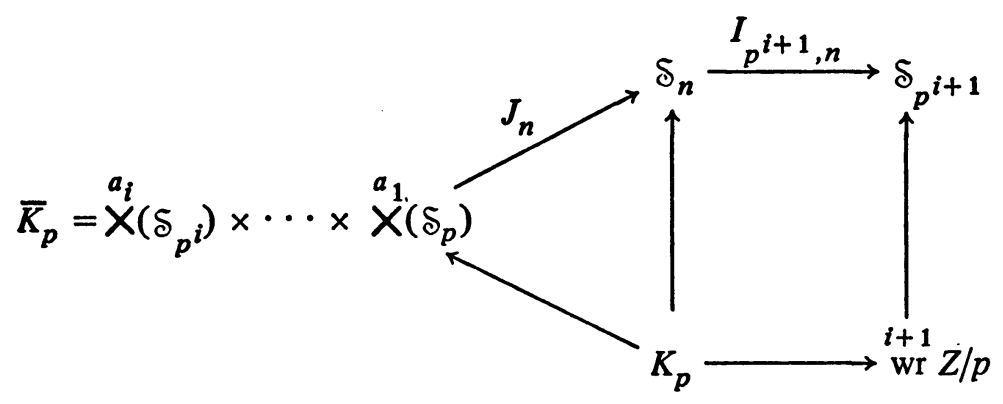

THEOREM F. (1) $I_{p^{*+1}, n}^{*}$ is surjective.

(2) $J_{n}^{*}$ is injective.

(3) $v \in$ Image $J_{n}^{*}$ if and only if there exists a $u \in H^{*}\left(\mathcal{S}_{p^{\prime+1}}\right)$ such that

$$
\begin{aligned}
& \left(I_{p^{i+1, n}} \circ J_{n}\right)^{*}(u)=v \\
& \quad=\sum \delta\left\langle u_{i, 1}, \ldots, u_{i, a_{i}}\right\rangle \otimes \cdots \otimes \delta\left\langle u_{1,1} \cdots u_{i, a_{1}}\right\rangle \in H^{*}\left(\bar{K}_{p}\right)
\end{aligned}
$$

with $u_{t, r} \in H^{*}\left(\mathcal{S}_{p^{\prime}}\right)$ for each $r$.

IMPORTANT EXAMPLE. Let $n=2 p^{i}$. We have

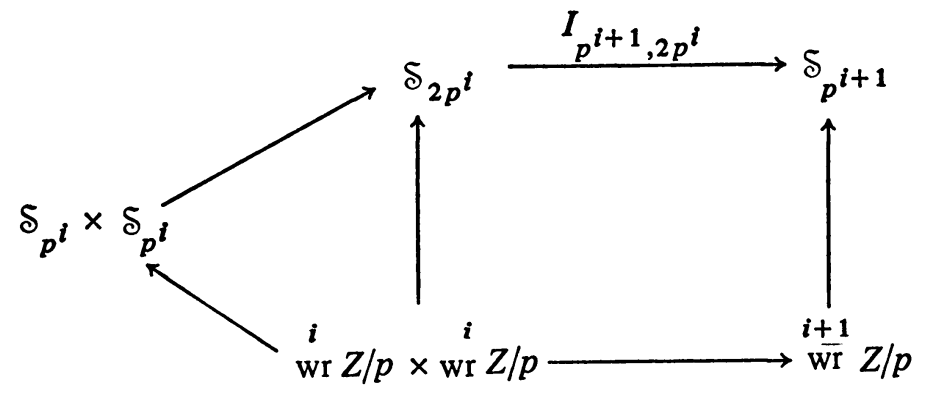

Recall the definition of $A_{k, i}$ and $B_{k, i}$ (see example (i) after Theorem B). Then $I_{p^{i+1}, 2 p^{\prime}}^{*}\left(A_{k, i+1}\right)=A_{k, i} \otimes 1+1 \otimes A_{k, i}=\mathcal{S}\left\langle A_{k, i}, 1\right\rangle$ for $1 \leqslant k \leqslant p^{i}$, while for 
$p^{i}<k \leqslant p^{i+1}, I_{p^{i+1}, 2 p^{i}}^{*}\left(A_{k, i+1}\right)=A_{k, i}^{\prime} \otimes 1+1 \otimes A_{k, i}^{\prime}$ where $A_{\mathrm{k}, \mathrm{i}}^{\prime}$ is expressible in terms of $A_{r, i}$ and $B_{r, i}$ for $r<p^{i}$.

$$
I_{p^{i+p, 2 p^{i}}}^{*}\left(B_{k, i+1}\right)=\sum_{n+m=k} B_{n, i} \otimes B_{m, i}=\sum_{n=0}^{p^{\prime}} \delta\left\langle B_{n, i}, B_{2 p^{i}-n, i}\right\rangle,
$$

where $0 \leqslant n, m \leqslant p^{i}, 0 \leqslant k \leqslant 2 p^{i}$, and $B_{0, i}=1$. Similar restrictions occur on the other detecting groups. Thus the natural inclusions $\delta_{n} \rightarrow \delta_{n+1}$ $\rightarrow \cdots \rightarrow \operatorname{dir} \lim \delta_{n}$ are easily analyzed. Clearly

$$
\delta_{p^{\prime}} \rightarrow \delta_{p^{\prime+1}} \rightarrow \cdots \rightarrow \operatorname{dir} \lim \delta_{p^{\prime}}
$$

is a cofinal direct limit and we have $H^{*}\left(\operatorname{dir} \lim \delta_{n}\right) \cong H^{*}\left(\operatorname{dir} \lim \delta_{p^{\prime}}\right) \cong$ inv $\lim H^{*}\left(\delta_{p^{i}}\right)$. Notice Theorem $\mathrm{F}$ implies inv $\lim H^{t}\left(\delta_{p^{i}}\right)$ is attained for each $t$ at a finite stage.

Recall the theorem stated in the introduction that ties $\operatorname{dir} \lim B_{\delta_{n}}$ to $Q\left(S^{0}\right)=\operatorname{dir} \lim \Omega^{n} S^{n}$. Furthermore, if $G_{n}$ is the set of homotopy equivalences of $S^{n-1}$ then $G=\operatorname{dir} \lim G_{n}$ is homotopy equivalent to the union of the +1 and -1 components of $Q\left(S^{0}\right)$. Thus $\operatorname{dir} \lim B_{\delta_{n}}$ properly interpreted is a model for $G$ and we have:

$$
\text { inv } \lim H^{*}\left(\delta_{p^{i}}\right) \cong H^{*}\left(Q\left(S^{0}\right)_{0}\right) \cong H^{*}(S G)
$$

as algebras. Thus $H^{*}(S G)$ can be identified with "infinite symmetric sums" in the $\mathcal{W}_{i}$ algebras with the proper identifications; i.e., $\delta\left\langle Q_{j, i}, 1, \ldots\right\rangle \leftrightarrow$ $\delta\left\langle Q_{j-1, i-1}, \ldots, Q_{j-1, i-1}, 1, \ldots\right\rangle$. The $\mathscr{Q}(p)$ action on $H^{*}(S G)$ restricts to that on $B_{\delta_{p^{\prime}}}$ for each $i$ and there is a unique action which has this property. Theorem $E$ describes the restriction of this action. Recall, [22] and [24], $H^{*}\left(\operatorname{dir} \lim \delta_{p^{\prime}}\right)$ is a Hopf algebra isomorphic to $H^{*}\left(Q\left(S^{0}\right)_{0}\right)$ with the coalgebra product on $H^{*}\left(\operatorname{dir} \lim \delta_{p^{\prime}}\right)$ induced by the inclusions $\delta_{p^{\prime}} \times \delta_{p^{\prime}} \rightarrow$ $\delta_{2 p^{\prime}}$. Thus Theorem $\mathrm{F}$ gives the loop sum coalgebra map on $H^{*}\left(Q\left(S^{0}\right)_{0}\right)$.

As $Q\left(S^{0}\right)_{0}$ is an $H$-space it is possible to obtain integral information about $H^{*}(S G, Z, p)$ on $H^{*}\left(\mathcal{S}_{p^{\prime}}, Z, p\right)$ (see [14]). [2] gives a Hopf algebra Bockstein spectral sequence with

$$
\begin{aligned}
& E_{1} \cong H^{*}\left(\operatorname{dir} \lim \delta_{p^{i}}, Z / p\right), \\
& E_{\infty} \cong H^{*}\left(\operatorname{dir} \lim \delta_{p^{i}}, Z, p\right) / \text { Torsion. }
\end{aligned}
$$

Let $x, y \in \mathscr{W}_{j}$ and let

$$
L_{n j}\left(x: y_{n+1}, \ldots, y_{m}, 1, \ldots\right)=\delta\left\langle x L_{j}^{p-1}, \ldots, x L_{j}^{p-1}, y_{n+1}, \ldots, y_{m}, 1, \ldots\right\rangle
$$

and

$$
\begin{aligned}
& \underline{L}_{n j}\left(x: y_{n+1}, \ldots, y_{m}, 1, \ldots\right) \\
& \quad=\delta\left\langle x \underline{L}_{j} L_{j}^{p-2}, x L_{j}^{p-1}, \ldots, x L_{j}^{p-1}, y_{n+1}, \ldots, y_{m}, 1, \ldots\right\rangle
\end{aligned}
$$

where $y_{r} \neq x L_{j}^{p-1}$ or $x \underline{L}_{j} L_{j}^{p-2}$. Note a class in $H^{*}\left(\operatorname{dir} \lim \delta_{p^{i}}\right)$ may have 
more than one representation as $L_{n j}(\cdots)$ or $\underline{L}_{n j}(\cdots)$; for example,

$$
\mathcal{S}\left\langle x L_{j}^{p-1}, x L_{j}^{p-1}, y L_{j}^{p-1}, 1, \ldots\right\rangle=L_{2, j}(x: y, 1, \ldots)=L_{1, j}(y: x, 1, \ldots) \text {. }
$$

TheOREM G. Let $k_{j, \infty}^{*}=\operatorname{dir} \lim _{i} k_{j, i}^{*}$ and let $u \in H^{*}\left(\operatorname{dir} \lim \S_{p^{\prime}}\right)$ be a proper class. Then there exists a smallest positive integer $j$ such that $k_{j, \infty}^{*}(u) \neq 0$. Then $k_{j, \infty}^{*}(u)=\delta\left\langle x_{1}, \ldots, x_{m}, 1, \ldots\right\rangle$ and

(1) If some $x_{n}$ contains an odd number of $M_{g, j}$ factors or if $k_{j, \infty}^{*}(u)=$ $\underline{L}_{n, j}(\cdots)$ or $L_{n, j}(\cdots)$ for $n$ not divisible by $p$ then $u$ is in the image or domain of $\beta_{p}$.

(2) Let $r \geqslant 2$. If $d_{r-1}(v)=u$ in $E_{r-1}$ of the Bockstein spectral sequence and $k_{j, \infty}^{*}(u)=\delta\left\langle x_{1}, \ldots, x_{m}, 1, \ldots\right\rangle$ with no $x_{n}$ containing an odd number of $M_{g, h}$ terms or the factor $\underline{L}_{j}$ then there exist $v^{\prime}$ and $u^{\prime}$ such that $d_{r}\left(v^{\prime}\right)=u^{\prime}$ where $k_{j, \infty}^{*}\left(u^{\prime}\right)=\delta\left\langle x_{1}, \ldots, x_{1}, \ldots, x_{m}, \ldots, x_{m}, 1, \ldots\right\rangle+\Sigma u^{\prime \prime}$. Each $x_{h}$ appears $p$ times in $\delta\left\langle x_{1}, \ldots, x_{1}, \ldots, x_{m}, \ldots, x_{m}, 1, \ldots\right\rangle$ and each $u^{\prime \prime}=$ $\delta\left\langle x_{1}, \ldots, x_{t}, 1, \ldots\right\rangle$ with $t<p m$.

COROLLARY 1. Let $r \geqslant 2$ then

$$
d_{r}\left(L_{p^{r-1}, j}(x: 1, \ldots)\right)=L_{p^{r-1}, j}(x: 1, \ldots)
$$

where $x$ satisfies the same conditions as the $x_{n}$ 's in (2) of Theorem G.

Let $R_{i}$ be the inclusion $\delta_{p^{\prime}} \rightarrow \operatorname{dir} \lim \delta_{p^{\prime}}$ then $R_{i}^{*}$ gives the Bockstein structure of $H^{*}\left(\delta_{p^{\prime}}, Z, p\right)$.

Corollary 2. $Q_{j, i} \in H^{*}\left(\delta_{p^{i}}, Z, p\right)$ has order $p^{j+1}$.

ExAMPLES. (i) $L_{p^{\prime}, j}\left(M_{1, j} L_{j} L_{j}^{p-3}: 1, \therefore\right)$ is a class of order $p$ in $H^{*}(S G, Z$, $p$ ), while $L_{p^{\prime} j}\left(M_{1, j} M_{2, j} L_{j}^{p-3}: 1, \ldots\right)$ is a class of order $p^{r+1}$.

(ii) $\left(B_{6}, \delta\left\langle Q_{1,2}, Q_{1,2}, 1\right\rangle, 0\right) \in H^{24}\left(\mathcal{S}_{27}, Z, 3\right)$ has order 9 .

Finally the results of this paper have an application to cobordism theory. Although [3], [13] and [18] completely compute the PL and TOP cobordism ring at the prime 2 , the odd case still has unanswered questions, notably the odd torsion in $\Omega^{\mathrm{PL}}$. Using results of [3], [15], [26], [27], [32], [34], [37], [38], [39] and this paper one may calculate the $E^{2}$ term of the Adams spectral sequence converging to $\Omega^{\mathrm{PL}} \otimes Z_{(p)}$. Current joint work with H. Ligaard, J. P. May and R. J. Milgram computes this $E^{2}$ term and gives infinite families of nontrivial differentials of all orders in the spectral sequence.

II. The embedding and the detecting family.

2.1. Definition. Let $K$ be a finite group and $L$ a subgroup of $\delta_{n}$ then $K$ wr $L$ is defined to be the group whose elements are

$$
\{(f, g): f \text { is a mapping of }(1,2, \ldots, n) \text { into } K, g \in L\}
$$

and whose multiplication is given by $(f, g)\left(f^{\prime}, g^{\prime}\right)=\left(f f_{g}^{\prime}, g g^{\prime}\right)$, where $f_{g}(g(i))=$ $f(i)$ and $f f^{\prime}(i)=f(i) f^{\prime}(i)$. 
2.2. Definition. Let $X$ be a space and $\left\{A_{i}\right\}$ a collection of subspaces of $X$. $\left\{A_{i}\right\}$ is a $Z / p$ cohomology detecting family for $X$ if the inclusion map $H^{*}(X) \rightarrow$ $\Pi H^{*}\left(A_{i}\right)$ is an injection.

2.3. Lemma. Let $K_{p}$ be a $p$-Sylow subgroup of $K$, then the transfer $t\left(K, K_{p}\right)$ : $H^{*}\left(K_{p}\right) \rightarrow H^{*}(K)$ is an epimorphism and the inclusion $i\left(K_{p}, K\right): H^{*}(K) \rightarrow$ $H^{*}\left(K_{p}\right)$ is a monomorphism whose image consists of stable elements of $H^{*}\left(K_{p}\right)$. Furthermore we have the direct sum decomposition $H^{*}\left(K_{p}\right) \cong \operatorname{Im} i\left(K_{p}, K\right) \oplus$ $\operatorname{Ker} t\left(K, K_{p}\right)$.

Proof. See [5, Chapter XII, p. 257] for the definition of stable and p. 259 for a proof of the lemma.

Recalling that a $p$-Sylow subgroup of $\delta_{p^{\prime}}$ is isomorphic to $\mathrm{wr}^{i} Z / p$, [6] gives

2.4. CoRollary. If $\left\{A_{j}\right\}$ is a $Z / p$ detecting family for $\mathrm{wr}^{i} Z / p$ then it is one for $\delta_{p^{\prime}}$ also.

2.5. Definition. Let $G$ be a finite group of order $n$. Then the adjoint representation $A: G \rightarrow \delta_{n}$ is defined as follows: Let $A(g)$ be the permutation $\left\{g_{i} \mapsto g g_{i}\right\}$ where $\delta_{n}$ is thought of as the permutations on the $n$ elements of $G$.

The adjoint representation is obviously a monomorphism and includes $G$ in $\delta_{n}$. Let $G=\times^{i} Z / p$, then the adjoint representation of $\times^{i} Z / p$ in $\delta_{p^{i}}$ is clearly equivalent to the map $k_{i, i}: X^{i} Z / p \rightarrow \delta_{p^{\prime}}$ defined in $\S$ I. (The two maps differ by at most a reordering of the elements of $\times^{i} Z / p$; that is, an inner automorphism of $\delta_{p^{\prime} \text {.) }}$

Again considering $\delta_{p^{\prime}}$ as the permutations on the set $\Pi^{i} Z / p$ the map $I_{i-1}$ : $X_{m=1}^{p}\left(\delta_{p^{\prime-1}}\right)_{m} \rightarrow \delta_{p^{\prime}}$ defined in the introduction is realized by letting $\left(\delta_{p^{i-1}}\right)_{m}$ permute the set $\Pi^{i-1} Z / p \times\{m\}$ contained in $\Pi^{i} Z / p$.

Note that under the specific embeddings $k_{i, i}$ and $I_{i-1}$ the subgroup $\times^{i-1} Z / p \times\{0\} \rightarrow \times^{i} Z / p \rightarrow{ }^{k_{i, i}} \delta_{p^{\prime}}$ is contained in the subgroup $\times_{m=1}^{p}\left(\delta_{p^{i-1}}\right)_{m} \rightarrow^{I_{i-1}} \delta_{p^{\prime}}$. Any $p$-Sylow subgroup of $X_{m=1}^{p}\left(\delta_{p^{i-1}}\right)_{m}$ that contains $X^{i-1} Z / p \times\{0\}$ is isomorphic to $X_{m=1}^{p}\left(\mathrm{wr}^{i-1} Z / p\right)_{m}$. Then $\times_{m=1}^{p}\left(\mathrm{wr}^{i-1} Z / p\right)_{m}$ and $\times^{i} Z / p$ generate a $p$-Sylow subgroup of $\delta_{p^{\prime}}$ which must be isomorphic to $\mathrm{wr}^{i} Z / p$. Thus we have the following commutative diagram with the above mentioned inclusions:

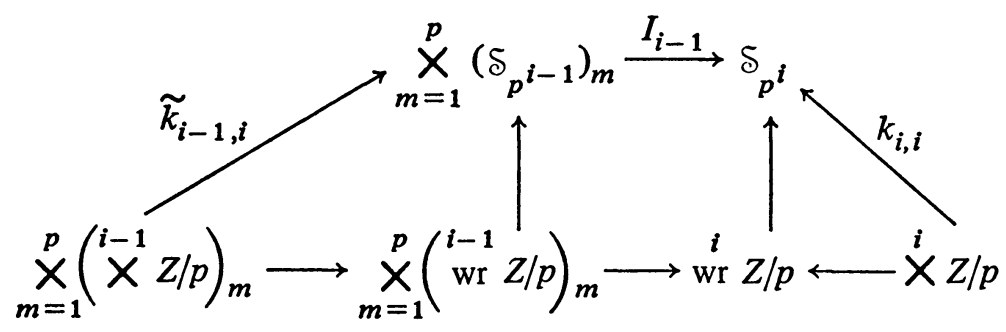


where $\tilde{k}_{i-1, i}=\times{ }_{m=1}^{p}\left(k_{i-1, i-1}\right)_{m}$. The specific form of $k_{i, i}$ and $\tilde{k}_{i-1, i}$ guarantees $X_{m=1}^{p}\left(X^{i-1} Z / p\right)_{m}$ factors through $X_{m=1}^{p}\left(w r^{i-1} Z / p\right)_{m}$.

More generally if $I_{m_{1}, \ldots, m_{n}}: \mathcal{S}_{p^{m_{1}}} \times \cdots \times \mathcal{S}_{p^{m_{n}}} \rightarrow \mathcal{S}_{p^{\prime}}$ is defined by letting $\delta_{p_{r}}$ permute the $p^{m_{r}}$ letters $\left(p^{m_{1}}+\cdots+p^{m_{r-1}}+1, \ldots, p^{m_{1}}+\cdots+p^{m_{r}}\right)$ then the map $I_{m_{1}, \ldots, m_{n}} \circ\left(\prod_{r=1}^{n} k_{m_{r}, m_{r}}\right)$ includes $\Pi_{r=1}^{n}\left(X^{m_{r}} Z / p\right)$ in $\delta_{p^{\prime}}$.

If $m_{1}=m_{2}=\cdots=m_{p^{i-j}}=j$ then $\Pi_{r=1}^{p^{i-j}}\left(X^{j} Z / p\right) \rightarrow \mathcal{S}_{p^{\prime}}$ has the form

$$
k_{j, i}=I_{j, \ldots, j} \circ \prod_{r=1}^{p^{\prime-j}}\left(k_{j, j}\right)_{r}: \stackrel{p^{i-j}}{\times}(\stackrel{j}{\times} Z / p) \rightarrow \delta_{p^{\prime}} .
$$

2.6. Definition. Let $T_{j, i}=\times^{p^{i-j}}\left(X^{j} Z / p\right)$. Let $k_{j, i}: T_{j, i} \rightarrow \delta_{p^{\prime}}$ be the above inclusion. Then $T_{j, i}$ is called a totally symmetric detecting group.

Notice $T_{j, i}$ and $k_{j, i}$ are defined for $1<j<i$. The following lemmas are established in the proofs of Theorems A through D:

2.7. LEMMA. The set $\left\{I_{m_{1}, \ldots, m_{n}} \circ\left(\Pi_{r=1}^{n}\left(k_{m_{r}, m_{r}}\right)\right): \Pi_{r=1}^{n} \times m_{r}(Z / p) \rightarrow \mathcal{S}_{p^{\prime}}\right\}$ forms a $Z / p$ detecting family for $\mathcal{S}_{p^{\prime}}$.

2.8. LEMMA. The totally symmetric detecting groups $T_{j, i}, 1<j<i$, detect a set of multiplicative generators for $H^{*}\left(\delta_{p^{\prime}}\right)$. (This is the first part of Theorem D.)

2.9. LeMMA. In $Z / p$ cohomology, $\operatorname{Ker} k_{i, i}^{*} \cap \operatorname{Ker} I_{i-1}^{*}=0$.

These lemmas may be proved directly using [27], induction on $i$, and 3.1.

We now examine the normalizers of the detecting subgroups in $\delta_{p^{\prime}}$. Consider $k_{i, i}: T_{i, i} \rightarrow \mathcal{S}_{p^{\prime}}$. Let $a_{r} \in \mathcal{S}_{p^{\prime}}$ generate $k_{i, i}\left(0 \times 0 \times \cdots \times(Z / p)_{r}\right.$ $\times \cdots \times 0)$ and let $N_{i}$ be the normalizer of $k_{i, i}\left(T_{i, i}\right)$ in $\delta_{p^{\prime}}$. Define a homomorphism $\psi: N_{i} \rightarrow \mathrm{GL}(i, Z / p)$ as follows: If $x \in N_{i}$ then $x a_{r} x^{-1}=$ $a_{1}^{s_{1, r}} a_{2}^{s_{2, r}} \cdots a_{i}^{s_{i, r}}$. Then let $\psi(x)$ be the matrix whose $(m, n)$ th entry is $s_{m, n}$. Clearly $\psi(x)$ is nonsingular.

2.10. Proposition. The sequence $1 \rightarrow k_{i, i}\left(T_{i, i}\right) \rightarrow N_{i} \rightarrow^{\psi} \mathrm{GL}(i, Z / p) \rightarrow 1$ is exact.

Proof. Preceding $k_{i, i}$ by any automorphism $\varphi: T_{i, i} \rightarrow T_{i, i}$ is just a reordering of the underlying set of $T_{i, i}$. This reordering, considered as an element of $\S_{p^{i}}$, conjugates $k_{i, i}$ to $k_{i, i} \circ \varphi$. This implies $\psi$ is onto. The remainder of the proposition follows trivially.

For $x \in \mathcal{S}_{p^{\prime}}$ the homomorphism ad $\left.\operatorname{ad}_{x}: T_{i, i}\right) \rightarrow H^{*}\left(x T_{i, i} x^{-1}\right)$ is induced by the inner automorphism $y \rightarrow x y x^{-1}$. Let $E=\sum_{m=1}^{i} a_{m} e_{m}$ and $B=$ $\sum_{m=1} a_{m}^{\prime} b_{m}$ in $H^{*}\left(T_{i, i}\right)$ then it follows directly from the definition of $\psi$ that

2.11. Proposition. For $x \in N_{i}, \operatorname{ad}_{x}(E)=\psi(x) E$ and $\operatorname{ad}_{x}(B)=\psi(x) B$.

Since $\operatorname{ad}_{x}$ is a ring homomorphism 2.11 determines $\operatorname{ad}_{x}$ on all of $H^{*}\left(T_{i, i}\right)$. 
Since the $p$ th power homomorphism, $a \mapsto a^{p}$, is the identity on $Z / p$ we have $P\left(x p, \ldots, x_{i}^{p}\right)=\left(P\left(x_{1}, \ldots, x_{i}\right)\right)^{p}$ for all polynomials $P$. This fact and direct computation yield

2.12. Proposition. ad ${ }_{x}$ operates on the classes $L_{i}, Q_{j, i}, M_{j, i}, \underline{L}_{i}$ via multiplication by the determinant function.

2.13. CoROLlaRY. The algebra $\mho_{i}$ is contained in $H^{*}\left(T_{i, i}\right) \mathrm{GL}(i, Z / p)$.

2.14. LEMMA. If $G$ is a finite group, $K$ a subgroup, and $N_{K, G}$ the normalizer of $K$ in $G$ then the image of $H^{*}(G)$ in $H^{*}(K)$ is contained in $H^{*}(K)^{N_{K, G}}$.

Proof. Any inner automorphism of $G$ induces the identity on $H^{*}(G)$. Hence we have the following commutative diagram:

$$
\begin{array}{ccc}
H^{*}(G) & \stackrel{\text { id }}{\rightarrow} & H^{*}(G) \\
i(K, G) \downarrow & & \downarrow i\left(x K x^{-1}, G\right) \\
H^{*}(K) & \stackrel{\text { ad }_{x}}{\rightarrow} & H^{*}\left(x K x^{-1}\right)
\end{array}
$$

Allowing $x$ to run through $N_{K, G}$ gives the lemma.

2.15. Corollary. Let $u \in H^{*}\left(\mathcal{S}_{p^{i}}\right)$ then $k_{i, i}^{*}(u) \in H^{*}\left(T_{i, i}\right) \mathrm{GL}(i, Z / p)$.

ProOF. Immediate from 2.10 and 2.14.

Let $N_{j, i}$ be the normalizer of $k_{j, i}: T_{j, i} \rightarrow \delta_{p^{\prime}}$ in $\delta_{p^{\prime}}$.

2.16. Proposition. The sequence

$$
1 \rightarrow \stackrel{p^{i-\jmath}}{\times} N_{j} \rightarrow N_{j, i} \stackrel{\varphi}{\rightleftarrows} \delta_{p^{i-\jmath}} \rightarrow 1
$$

is exact.

Proof. Both $N_{j, i}$ and $\times{ }^{p^{1-j}} N_{j}$ act on $T_{j, i}$ via conjugation. But $x \in N_{j, i}$ permutes the $p^{i-j}$ orbits of $\times^{p-j} N_{j}$. This gives a homomorphism $\varphi: N_{j, i} \rightarrow$ $\delta_{p^{\prime-j}}$ which is clearly onto and has an obvious section $\psi$. Notice $\psi\left(\varphi(x)^{-1}\right) \cdot x$ $\in \times^{p^{i-j}} N_{j}$ as $\psi\left(\varphi(x)^{-1}\right) \in N_{j, i}$ and $\psi\left(\varphi(x)^{-1}\right) \cdot x \in \times^{p^{i-j}} S_{p}$. The proposition follows.

Let $N_{m_{1}, \ldots, m_{n}}$ be the normalizer of $I_{m_{1}, \ldots, m_{n}}\left(\Pi_{r=1}^{n}\left(k_{m_{r}, m_{r}}\right)\right): \Pi_{r=1}^{n}\left(X^{m_{r}} Z / p\right)$ $\rightarrow \mathcal{S}_{p^{\prime}}$ in $\mathcal{S}_{p^{\prime}}$ and let $\mathcal{S}_{\left(m_{1}, \ldots, m_{n}\right)}$ be the subgroup of $\mathcal{S}_{n}$ generated by the transpositions $(a, c)$ where $m_{a}=m_{c}$. Minor modification of 2.16 yields the following three propositions.

2.17. Proposition. The sequence $1 \rightarrow \times_{r=1}^{n} N_{m_{r}} \rightarrow N_{m_{1}, \ldots, m_{n}} \rightleftarrows \delta_{\left(m_{1}, \ldots, m_{n}\right)}$ $\rightarrow 1$ is exact.

2.18. Proposition. Let $\bar{N}_{j}$ be the normalizer of $I_{j}: \times^{p^{\prime-j}} S_{p^{\prime}} \rightarrow \delta_{p^{\prime}}$ in $\delta_{p^{\prime}}$. Then the sequence $1 \rightarrow \times{ }^{p^{\prime-j}} \delta_{p^{\prime}} \rightarrow \bar{N}_{j} \rightleftarrows \delta_{p^{\prime-j}} \rightarrow 1$ is exact. 
2.19. PROPOSITION. Let $\bar{N}_{m_{1}, \ldots, m_{n}}$ be the normalizer of $I_{m_{1}, \ldots, m_{n}}: \times_{r=1}^{n} \delta_{m_{r}}$ $\rightarrow \delta_{p^{\prime}}$ in $\delta_{p^{\prime}}$. Then the sequence $1 \rightarrow X_{r=1}^{n} \delta_{m_{r}} \rightarrow \bar{N}_{m_{1}, \ldots, m_{n}} \rightleftarrows \mathcal{\delta}_{\left(m_{1}, \ldots, m_{n}\right)} \rightarrow$ 1 is exact.

2.20. LEMMA. If $G$ is a finite group and $K$ a subgroup then $i(K, G)^{*} t(G$, $K)=\sum_{x \in G / K^{\prime}} t_{x} i_{x} \operatorname{ad}_{x}$ where $\operatorname{ad}_{x}: H^{*}(K) \rightarrow H^{*}\left(x K x^{-1}\right)$ is the homomorphism induced by $y \mapsto x y x^{-1}$ for $y \in K, i_{x}$ is the inclusion map $H^{*}\left(x K x^{-1}\right) \rightarrow$ $H^{*}\left(x K x^{-1} \cap K\right)$ and $t_{x}$ is the transfer $H^{*}\left(x K x^{-1} \cap K\right) \rightarrow H^{*}(K)$.

Proof. [5, XII. 9.1, p. 257].

2.21. Proposition. If $K$ is a proper subgroup of $\times^{m} Z / p$ then the transfer $t$ : $H^{*}(K) \rightarrow H^{*}\left(X^{m} Z / p\right)$ is zero.

\section{Proof. [4, I.2.1].}

III. Some properties of $\mathcal{Q}(p)$ and the proof of Theorem $\mathbf{E}$. In this section we state facts about the Steenrod algebra needed to prove Theorems A through $\mathrm{D}$ and give a proof of Theorem $\mathrm{E}$.

First recall the construction of the Steenrod $p$ th powers ([31] gives the complete treatment and we quote it frequently in what follows). Let $X$ be a finite regular cell complex then we have the following spaces and maps:

$$
X^{p} \stackrel{j}{\rightarrow} W_{Z / p} \times_{Z / p} X^{p} \stackrel{1 \times \Delta}{\leftarrow} W_{Z / p} \times_{Z / p} X=B_{Z / p} \times X
$$

where $j$ is the inclusion and $\Delta$ is the diagonal map. Given any $u \in H^{*}(X)$ there exists a unique natural class $\mathscr{P}(u)$ in $H^{*}\left(W_{Z / p} \times_{z / p} X^{p}\right)$ such that:

(1) $j^{*}(\mathcal{P}(u))=u \otimes \cdots \otimes u=u^{\otimes p}$.

(2) $(1 \times \Delta)^{*}(\mathscr{P}(u))$ in $H^{*}\left(B_{Z / p} \times X\right)$ can be expanded by the Künneth theorem. $(1 \times \Delta)^{*}(\mathscr{P}(u))=\sum w_{k} \otimes D_{k}(u)$ where $w_{k}$ generates $H^{k}(Z / p)$ and $D_{k}: H^{q}(X) \rightarrow H^{p q-k}(X)$ are homomorphisms which define the elements of $\mathbb{Q}(p)$.

(3) $\beta D_{2 k}(u)=D_{2 k-1}(u), \beta D_{2 k-i}(u)=0$ and $\beta D_{0}(u)=0$.

3.1. THEOREM [31]. If $z \in H^{*}\left(W_{Z / p} \times_{Z / p} X^{p}\right)$, then $z$ is of the form $z=t z_{1}+z_{2} \cdot \mathscr{P}\left(z_{3}\right)$ with $z_{1} \in H^{*}\left(X^{p}\right), z_{2} \in H^{*}\left(B_{z / p}\right)$ and $z_{3} \in H^{*}(X)$, where $t$ is the transfer. Furthermore the sequence

$$
H^{*}\left(X^{p}\right) \stackrel{t}{\rightarrow} H^{*}\left(W_{Z / p} \times_{Z / p} X^{p}\right) \stackrel{(1 \times \Delta)^{*}}{\rightarrow} H^{*}\left(B_{Z / p} \times X\right)
$$

is exact.

Proof. [31, VII. 4.1, p. 104 and VIII. 3.6, p. 126].

3.2. Definition [31]. Let $u \in H^{q}(X)$ then

$$
\begin{aligned}
\mathscr{P}^{j}(u) & =a_{j, q} D_{(q-2 j)(p-1)}(u), \\
\beta^{j}(u) & =a_{j, q} D_{(q-2 j)(p-1)-1}(u),
\end{aligned}
$$


where $a_{j, q}$ is a nonzero constant in $Z / p$ dependent on $j$ and $q$. If $k \neq(q-2 j)(p$ $-1)$ or $(q-2 j)(p-1)-1$ for some $j$ then $D_{k}(u)=0$.

3.3. Proposition. If $q$ is even, say $q=2 n$, then $a_{j, 2 n}=(-1)^{j+n}$.

Proof. Follows directly from [31, VII. 6.1 and VII. 6.3] (note correction of the formula in VII. 6.1 on the first page of the appendix to [31]).

The following is well known:

3.4. LEMMA. I. Let $p$ be a prime and $a=\sum_{i=0}^{m} a_{i} p^{i}, c=\sum_{i=0}^{m} c_{i} p^{i}\left(0 \leqslant a_{i}\right.$, $\left.c_{i} \leqslant p-1\right)$. Then

$$
\left(\begin{array}{l}
c \\
a
\end{array}\right) \equiv \prod_{i}\left(\begin{array}{l}
c_{i} \\
a_{i}
\end{array}\right)(\bmod p)
$$

II. $\mathscr{P}^{j}(e)=0$ for all $j>0$.

III. $\mathcal{P}^{j}\left(b^{k}\right)=\left(\begin{array}{c}k \\ j\end{array}\right) b^{k+(p-1) j}$.

IV. (Cartan formula) $\mathscr{\rho}^{j}(u v)=\sum_{m+n=j}{\rho^{m}}^{m}(u)^{\rho^{n}}(v)$.

V.

$$
\mathscr{P}^{j}\left(b^{p^{m}}\right)=\left(\begin{array}{c}
p^{m} \\
j
\end{array}\right) b^{p^{m}+(p-1) j}= \begin{cases}b^{p^{m}} & \text { if } j=0, \\
b^{p^{m+1}} & \text { if } j=p^{m} \\
0 & \text { otherwise. }\end{cases}
$$

Proof. [31, see I.2.6, V. 1, VII. 2.2 and VI. 2.3].

The proof of Theorem $\mathrm{E}$ follows from direct calculation and Lemma 3.4. Note: To prove relation (4) of Theorem E, just expand $\rho^{p^{k-1}}\left(Q_{k, i} L_{i}^{p-1}\right)$.

IV. Symmetric products and image $k_{i, i}^{*}$. In this chapter we summarize results of [17] which give $H^{*}\left(\mathcal{S}_{n}\right)$ as $Z / p$ vector spaces and give an upper bound on the size of image $k_{i, i}^{*}$.

Recall the monomial $\mathscr{P}^{I}=\beta^{\varepsilon_{k} \odot s_{k}} \cdots \beta^{\varepsilon_{1} \rho s_{1}} \in \mathbb{Q}(p)$ is called admissible if $s_{i} \geqslant p s_{i-1}+\varepsilon_{i-1}$ for each $i \geqslant 1$, and the excess of $\mathscr{P P}^{I}=2 s_{k}+\varepsilon_{k}$ $\sum_{j=1}^{k-1}\left(2 s_{j}(p-1)+\varepsilon_{j}\right)$. The excess of any admissible monomial is nonnegative. Let $\mathbb{Q}(p)_{n}$ be the subvector space of $\mathbb{Q}(p)$ spanned by those monomials of excess $<n$.

Let $\mathrm{SP}^{k}\left(S^{2 n}\right)$ be the $k$ symmetric product of $S^{2 n}$ (see [17] for the definition and properties of the symmetric products of a space).

4.1. THEOREM [17]. (1) $H_{*}\left(\operatorname{SP}^{k}\left(S^{2 n}\right)\right)=\sum_{m=1}^{k} H_{*}\left(\operatorname{SP}^{m}\left(S^{2 n}\right), \operatorname{SP}^{m-1}\left(S^{2 n}\right)\right)$.

(2) $\Re\left(S^{2 n}, Z / p\right)=\sum_{m=1}^{\infty} H_{*}\left(\operatorname{SP}^{m}\left(S^{2 n}\right), \operatorname{SP}^{m-1}\left(S^{2 n}\right)\right)$ is isomorphic to $H_{*}(K(Z, 2 n))$.

There is a bigrading of $\Re\left(S^{2 n}, Z / p\right)$ given by

$$
\Re_{i, m}\left(S^{2 n}, Z / p\right)=H_{i}\left(\operatorname{SP}^{m}\left(S^{2 n}\right), \operatorname{SP}^{m-1}\left(S^{2 n}\right)\right) .
$$

(3) For $\Re\left(S^{2 n}, Z / p\right)$ the generators $q_{I}$ in homology are in 1-1 correspondence with admissible monomials $\mathscr{P}^{I}=\beta^{\varepsilon_{i}} \mathcal{P} s_{i} \cdots \beta^{\varepsilon_{1}} \mathcal{P} s_{1}$ in $\mathbb{Q}(p)_{2 n}$ and the bidegree 
of this generator is $\left(\left|\mathscr{P}^{I}\right|+2 n, p^{i}\right)$. Moreover $\left\langle q_{I}, \mathscr{P}^{I}(i)\right\rangle=1$ under the isomorphism in (2).

Proof. [17].

REMARKS. (1) is due to N. E. Steenrod. [8] and [21] also studied (1) and (2).

The next theorem follows from the fact that the singular locus of $\left(S^{2 n}\right)^{p^{\prime}}$ under $\delta_{p^{i}}$ has dimension $2 n\left(p^{i}-1\right)$.

4.2. ThEOREM [17]. For $k<2 n-1, H^{k}\left(\mathcal{S}_{p^{i}}\right) \cong H_{2 n\left(p^{\prime}\right)-k}\left(\operatorname{SP}^{p^{\prime}}\left(S^{2 n}\right)\right)$.

Since $H_{j}\left(\operatorname{SP}^{p^{\prime}}\left(S^{2 n}\right)\right) \cong H_{j}\left(\operatorname{SP}^{p^{\prime}}\left(S^{2 n}\right), \operatorname{SP}^{p^{\prime}-1}\left(S^{2 n}\right)\right)$ for $j>2 n\left(p^{i}-1\right)+1$ we may identify $H^{k}\left(\mathcal{S}_{p^{i}}\right)$ with elements in $\Re\left(S^{2 n}, Z / p\right)$ of bidegree $\left(2 n\left(p^{i}\right)\right.$ $\left.-k, p^{i}\right)$. Thus for $k<2 n-1$ classes in $H^{k}\left(\delta_{p^{\prime}}\right)$ correspond to classes $\Sigma a$; with each $a \in \mathcal{R}\left(S^{2 n}, Z / p\right)$ having bidegree $\left(-, p^{i}\right)$. This gives $H^{k}\left(\delta_{p^{\prime}}\right)$ as $Z / p$ vector spaces. Recall there are two types of classes in $R\left(S^{2 n}, Z / p\right)$ having bidegree $\left(-, p^{i}\right)$ :

(1) $a$ corresponds to $\mathscr{P} I_{i}$ of bidegree $\left(\left|\mathscr{P}^{I_{i}}\right|+2 n, p^{i}\right)$,

(2) $a=\Pi b_{k}$ where $b_{k}$ has bidegree $\left(-, p^{j}\right)$, for some $j<i$ and occurs in $H_{*}\left(\operatorname{SP}^{p^{j}}\left(S^{2 n}\right), \operatorname{SP}^{p^{j}-1}\left(S^{2 n}\right)\right)$.

On the other hand the multiplication map $M: \operatorname{SP}^{p^{\prime-1}}\left(S^{2 n}\right) \times \cdots \times$ $\operatorname{SP}^{p^{i-1}}\left(S^{2 n}\right) \rightarrow \operatorname{SP}^{p^{i}}\left(S^{2 n}\right)$ and 4.2 give a map $m: \otimes^{p} H^{*}\left(\delta_{p^{i-1}}\right) \rightarrow H^{*}\left(\delta_{p^{i}}\right)$.

4.3. LEMMA [21]. $m$ is the transfer map induced by the inclusion

$$
I_{i-1}: \stackrel{p}{\times} \delta_{p^{i-1}} \rightarrow \delta_{p^{i}} .
$$

PROOF. [21].

4.4. LeMMA. Let $u \in H^{*}\left(\mathscr{S}_{p^{i}}\right)$ correspond to $a \in \Re\left(S^{2 n}, Z / p\right)$. If $a$ is of type 2 then $k_{i, i}^{*}(u)=0$.

Proof. Suppose $a$ is of type 2 then $a$ is in the image of $M_{*}$. By $4.3, u$ is in the image of the transfer $t: H^{*}\left(X^{p} \mathcal{S}_{p^{i-1}}\right) \rightarrow H^{*}\left(\delta_{p^{i}}\right)$. But 3.1 implies $k_{i, i}^{*} t=0$. Hence $k_{i, i}^{*}(u)=0$.

Let $\mathcal{R}_{2 n\left(p^{\prime}\right)-k, p^{\prime}}^{\prime}\left(S^{2 n}, Z / p\right)$ be the subspace of $\mathscr{R}_{2 n\left(p^{\prime}\right)-k p^{i}}\left(S^{2 n}, Z / p\right)$ spanned by elements of type 1 . Then 4.4 yields:

4.5. THEOREM [17]. As $Z / p$ vector spaces

$$
\operatorname{dim}\left(\left(\text { image } k_{i, i}^{*}\right)_{k}\right) \leqslant \operatorname{dim}\left(\Re_{2 n\left(p^{\prime}\right)-k, p^{\prime}}^{\prime}\left(S^{2 n}, Z / p\right)\right) .
$$

V. The proof of Theorem A. We now proceed with the proof of Theorem A.

\subsection{LEMMA. $\mho_{i}$ is contained in image $k_{i, i}^{*}$.}

Proof. By induction on $i$. The lemma is classically true for $i=1$ and [4] proves the lemma for $i=2$. Assume $\mho_{i-1}$ is contained in image $k_{i-1, i-1}^{*}$. The next four lemmas establish 5.1. 
5.2. LEMMA. There exists $u \in H^{*}\left(\mathscr{S}_{p^{i}}\right)$ such that

$$
k_{i-1, i}^{*}(u)=\left(M_{i-2, i-1} M_{i-3, i-1} L_{i-1}^{p-3}\right)^{\otimes p} \in H^{*}\left(T_{i-1, i}\right) .
$$

Proof. Recall the following commutative diagram containing the construction of the Steenrod powers on $\mathcal{S}_{p^{i-1}}$ :

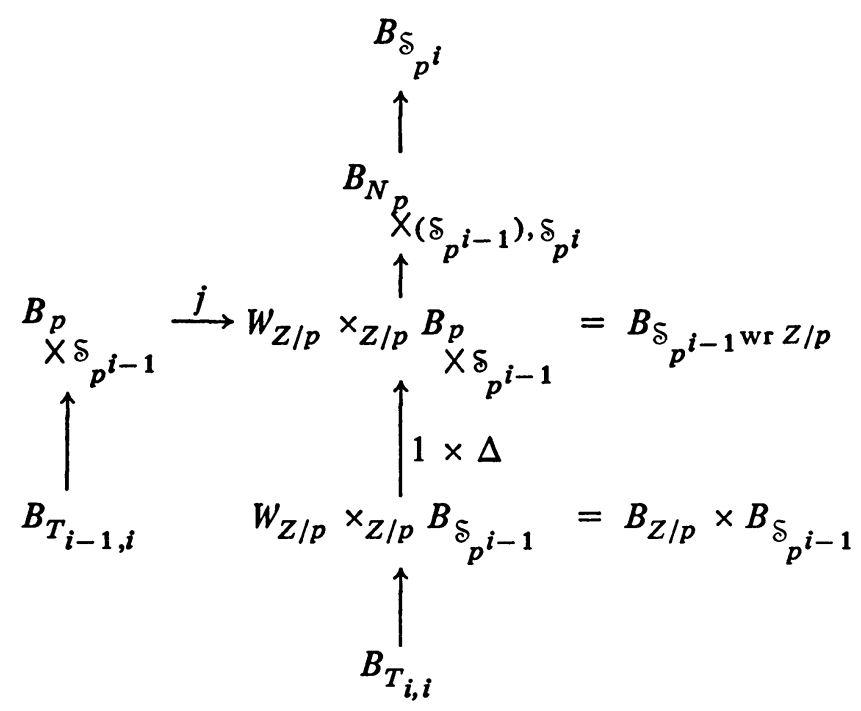

Of course the composition $B_{T_{i-1, i}} \rightarrow B_{\delta_{p^{\prime}}}$ is $B k_{i-1, i}$ and the composition $B_{T_{i, i}} \rightarrow B_{\delta_{p^{\prime}}}$ is $B k_{i, i}$.

Let $u^{\prime} \in H^{*}\left(\mathcal{\delta}_{p^{i-1}}\right)$ be such that $k_{i-1, i-1}^{*}\left(u^{\prime}\right)=M_{i-2, i-1} M_{i-3, i-1} L_{i}^{p-3}$ then $\mathscr{P}\left(u^{\prime}\right)=u^{\prime \prime} \in H^{*}\left(\mathcal{\delta}_{p^{\prime-1}} \mathrm{wr} Z / p\right)$. Let $A=\mathcal{S}_{p^{\prime-1}}$ wr $Z / p$. Then 2.20 gives

$$
i\left(A, \delta_{p^{i}}\right)^{*} t\left(\mathcal{S}_{p^{i}}, A\right)=\sum_{x \in \delta_{p^{i} / A}} t_{x} i_{x} \operatorname{ad}_{x}
$$

and we have the following commutative diagram:

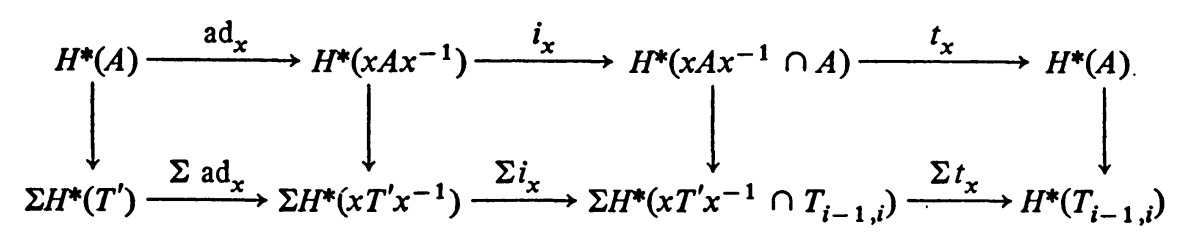

where $T^{\prime}$ runs through all inclusions $\times^{m} Z / p$ in $A$. (The last square commutes by 2.21 and $\left[31\right.$, V. 7.2], as $x T_{i-1, i} x^{-1} \subset A$ implies $x \in A$.)

Thus 2.16, 2.18 and 2.21 show

$$
k_{i-1, i}^{*} t\left(A, \delta_{p^{i}}\right)\left(u^{\prime \prime}\right)=\sum_{x}\left(M_{i-2, i-1} M_{i-3, i-1} L_{i-1}^{p-3}\right)^{\otimes p}
$$

where the sum runs over a coset representation $\bar{N}_{i-1}=N_{\times^{p} \delta_{p^{i}}-1, \delta_{p^{i}}} \bmod A$. As 
$A$ contains a $p$-Sylow subgroup of $\mathcal{S}_{p^{i}},\left[\bar{N}_{i-1}: A\right]=c \neq 0(\bmod p)$. Let $u=t\left(A, \delta_{p^{i}}\right)\left(c^{-1} u^{\prime \prime}\right)$; then $k_{i-1, i}^{*}(u)=\left(M_{i-2, i-1} M_{i-3, i-1} L_{i-1}^{p-3}\right)^{\otimes p}$.

5.3. LEMMA. There exists $u \in H^{*}\left(\complement_{p^{i}}\right)$ such that

$$
k_{i-1, i}^{*}(u)=\left(Q_{i-2, i-1}\right)^{\otimes p} \in H^{*}\left(T_{i-1, i}\right) \text {. }
$$

Proof. Identical to that of 5.2.

5.4. Lemma. There exists $u \in H^{*}\left(\mathcal{S}_{p^{i}}\right)$ such that $k_{i, i}^{*}(u)=M_{i-1, i} M_{i-2, i} L_{i}^{p-3}$.

Proof. Let $u^{\prime} \in H^{*}\left(\mathcal{\delta}_{p^{i-1}}\right)$ be such that $k_{i-1, i-1}^{*}\left(u^{\prime}\right)=$ $M_{i-2, i-1} M_{i-3, i-1} L_{i-1}^{p-3}$ and $u \in H^{*}\left(\delta_{p^{\prime}}\right)$ be such that $k_{i-1, i}^{*}(u)=$ $\left(M_{i-2, i-1} M_{i-3, i-1} L_{i-1}^{p-3}\right)^{\otimes p} \in H^{*}\left(T_{i-1, i}\right)$. Recall 3.1 implies image $k_{i, i}^{*}$ is contained in the $H^{*}(Z / p)$ module generated by image $(1 \times \Delta)^{*} \mathscr{P}$. A simple dimension check shows that the only classes in $H^{*}\left(\delta_{p^{i-1}} \mathrm{wr} Z / p\right)$ that could project to $k_{i-1, i}^{*}(u)$ are $\mathscr{P}\left(u^{\prime}\right)$ and $b_{1}^{x}+\mathscr{P}\left(u^{\prime}\right)$, where $x=\frac{1}{2} \operatorname{dimension}(u)$. By 2.15, $k_{i, i}^{*}(u)$ is $\mathrm{GL}(i, Z / p)$ invariant. As $\left(u^{\prime}\right)^{p}=0$ in $H^{*}\left(\delta_{p^{i-1}}\right)$ the class $b_{1}^{x}+\mathcal{P}\left(u^{\prime}\right)$ is not $\mathrm{GL}(i, Z / p)$ invariant (there cannot be a pure $b_{r}^{x}$ term in $(1 \times \Delta)^{*}\left(\mathscr{P}\left(u^{\prime}\right)\right)$ for $\left.r \geqslant 1\right)$. Hence $k_{i, i}^{*}(u)=(1 \times \Delta)^{*}\left(\mathscr{P}\left(u^{\prime}\right)\right)$. It is easy to see that dimension $\left(u^{\prime}\right)=2\left(p^{i-1}-p^{i-2}-p^{i-3}\right)=2 n$. Thus

$$
\begin{aligned}
& k_{i, i}^{*}(u)=(1 \times \Delta)\left(\mathscr{P}\left(u^{\prime}\right)\right)=\sum_{k} w_{k} \otimes D_{k}\left(M_{i-2, i-1} M_{i-3, i-1} L_{i-1}^{p-3}\right) \\
&=(-1)^{n}\left[\sum_{j} w_{(2 n-2 j)(p-1)} \otimes(-1)^{j} \mathcal{P}^{j}\left(M_{i-2, i-1} M_{i-3, i-1} L_{i-1}^{p-3}\right)\right. \\
&\left.+\sum_{j} w_{(2 n-2 j)(p-1)-1} \otimes(-1)^{j} \beta \mathcal{P}^{j}\left(M_{i-2, i-1} M_{i-3, i-1} L_{i-1}^{p-3}\right)\right] .
\end{aligned}
$$

Consider $M_{i-1, i} M_{i-2, i} L_{i}^{p-3}$. Expanding along the $e_{1}, b_{1}$ columns we have

$$
\begin{aligned}
M_{i-1, i} M_{i-2, i} L_{i}^{p-3}= & \sum_{\substack{A \\
B}}(-1)^{\varphi} b_{1}^{r}\left(A B C_{1} \cdots C_{p-3}\right) \\
& +\sum_{\substack{D \\
C_{k}}}(-1)^{\varphi} e_{1} b_{1}^{s}\left(D E C_{1} \cdots C_{p-3}\right)
\end{aligned}
$$

where $A$ runs over all $i-1 \times i-1$ minors of $M_{i-1, i}$ eliminating the $b_{1}^{p^{u}}$ $(0 \leqslant u \leqslant i-2)$ row and column, $B$ runs over all $i-1 \times i-1$ minors of $M_{i-2, i}$ eliminating the $b_{1}^{p^{v}}(0 \leqslant v \leqslant i-3$, or $v=i-1)$ row and column, $C_{k}$ $(k=1, \ldots, p-3)$ is any $i-1 \times i-1$ minor of $L_{i}$ eliminating the $b_{1}^{p^{2 k}}$ $\left(0 \leqslant z_{k} \leqslant i-1\right)$ row and column, $r$ satisfies the relation $\operatorname{dim}\left(M_{i-1, i} M_{i-2, i} L_{i}^{p-3}\right)=2 r+\operatorname{dim}(A)+\operatorname{dim}(B)+\sum_{k=1}^{p-3} \operatorname{dim}\left(C_{k}\right)$, and $\varphi \equiv$ $u+v+\sum_{k=1}^{p-3} z_{k}(\bmod 2)$ if $v \neq i-1$, and $\equiv(i-u)+\sum_{k=1}^{p-3} z_{k}(\bmod 2)$ if 
$v=i-1$. $D$ and $E$ are $i-1 \times i-1$ minors of $M_{i-1, i}$ and $M_{i-2, i}$ respectively with exactly one minor eliminating the $e_{1}$ row and column, the other eliminating a $b_{1}^{p^{\prime}}$ row and column.

If $C_{k}$ is the minor eliminating the $b_{1}^{p^{2}}$ row and column then $C_{k}=$ $\mathcal{\rho} m_{z_{k}}\left(L_{i-1}\right)$ where $m_{z_{k}}=p^{z_{k}}+p^{z_{k}+1}+\cdots+p^{i-2}\left(=0\right.$ if $\left.z_{k}=i-1\right)$.

Case 1. Suppose $v=i-1$. Then the minor of $M_{i-2, i}$ eliminating the $b_{1}^{p^{0}}$ row and column is $M_{i-2, i-1}$. If $A$ is an $i-1 \times i-1$ minor of $M_{i-1, i}$ eliminating the $b_{1}^{p^{u}}$ row and column and $A M_{i-2, i-1} \neq 0$ then $u \neq i-2$. Thus $A=\mathcal{P}^{j_{1}}\left(M_{i-3, i-1}\right)$ where $j_{1}=p^{u}+p^{u+1}+\cdots+p^{i-4}$ (if $u=i-3$ then $\left.j_{1}=0\right)$. Thus if $v=i-1$ we have

$$
\begin{aligned}
A B C_{1} \cdots C_{p-3} & \\
& =(-1) \rho^{0}\left(M_{i-2, i-1}\right) \rho^{j_{1}}\left(M_{i-3, i-1}\right) \rho^{m_{z_{1}}}\left(L_{i-1}\right) \cdots \rho^{m_{z_{p-3}}}\left(L_{i-1}\right) .
\end{aligned}
$$

Case 2. Suppose $0 \leqslant v \leqslant i-3$. Then $A=\mathscr{P}^{j_{1}}\left(M_{i-2, i-1}\right)$ where $j_{1}=p^{u}+$ $p^{u+1}+\cdots+p^{i-3}$ unless $u=i-2$ in which case $j_{1}=0$ and $B=$ $\rho^{j_{2}}\left(M_{i-3, i-1}\right)$ where $j_{2}=p^{v}+p^{v+1}+\cdots+p^{i-4}+p^{i-2}$ unless $v=i-3$ in which case $j_{2}=p^{i-2}$. Then we have

$$
A B C_{1} \cdots C_{p-3}=\mathcal{P}^{j_{1}}\left(M_{i-2, i-1}\right)^{\rho^{j_{2}}}\left(M_{i-3, i-1}\right) \mathcal{P m}^{m_{z_{1}}}\left(L_{i-1}\right) \cdots \mathcal{P}^{m_{z_{p-3}}}\left(L_{i-1}\right) .
$$

Note. In Case 1 we have terms involving $(-1) P^{0}\left(M_{i-2, i-1}\right) \mathscr{P}^{j_{1}}\left(M_{i-3, i-1}\right)$ and in Case 2 if $u=i-2$ we have terms involving $P^{0}\left(M_{i-2, i-1}\right)^{\mathcal{P j}_{2}}\left(M_{i-3, i-1}\right)$ but it is clear that $j_{1}$ can never equal $j_{2}$ in these cases.

Thus if $A B C_{1} \cdots C_{p-3} \neq 0$ we have written $A B C_{1} \cdots C_{p-3}$ uniquely as $\rho^{j_{1}}\left(M_{i-2, i-1}\right) \rho^{j_{2}}\left(M_{i-3, i-1}\right) \mathcal{\rho}^{m_{z_{1}}}\left(L_{i-1}\right) \cdots \mathcal{\rho}^{m_{z_{p-3}}}\left(L_{i-1}\right)$ for certain $j_{1}, j_{2}$, $m_{z}, \ldots, m_{z_{p-3}} .3 .4$ clearly shows if

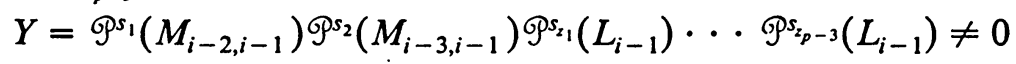

then $Y=A B C_{1} \cdots C_{p-3}$ for a suitable choice of $A, B, C_{1}, \ldots, C_{p-3}$ and is thus analyzed in Case 1 or Case 2 above.

Let $j=j_{1}+j_{2}+\sum_{k=1}^{p-3} m_{z_{k}}$. For both $v=i-3$ and $v<i-3$ it is trivial to see that $\varphi=j(\bmod 2)$. Hence the Cartan formula and the above facts yield the following decomposition of $M_{i-1, i} M_{i-2, i} L_{i}^{p-3}$ where the first sum runs over all integers $j$.

$$
\begin{aligned}
M_{i-1, i} M_{i-2, i} L_{i}^{p-3}= & \sum_{j} b_{1}^{(n-j)(p-1)} \otimes(-1)^{j} \mathscr{\rho}^{j}\left(M_{i-2, i-1} M_{i-3, i-1} L_{i-1}^{p-3}\right) \\
& +\sum_{\substack{E \\
C_{k}}}(-1)^{\phi} e_{1} b_{1}^{s} \otimes D E C_{1} \cdots C_{p-3} .
\end{aligned}
$$

Let $U=k_{i, i}^{*}(u)-(-1)^{n}\left(M_{i-1, i} M_{i-2, i} L_{i}^{p-3}\right) . U$ is clearly $\mathrm{GL}(i, Z / p)$ invariant. Any monomial term in $U$ must contain the factor $e_{1} e_{j}(j \neq 1)$ but as there is no monomial in $U$ with an $e_{2} e_{3}$ factor symmetry implies $U=0$. As 
$n=p^{i-1}-p^{i-2}-p^{i-3}$ we have

$$
k_{i, i}^{*}(u)=-M_{i-1, i} M_{i-2, i} L_{i}^{p-3} \text {. }
$$

This proves 5.4 .

Note. By keeping careful track of $D, E$, and $\beta\left(\mathscr{P}^{j_{1}}\left(M_{i-2, i-1}\right) \mathscr{P}^{j_{2}}\left(M_{i-3, i-1}\right)\right)$ it is possible to see directly that

$$
\begin{aligned}
\sum_{\substack{D \\
D_{k}}}(-1)^{\varphi} e_{1} b_{1}^{s} \otimes D E C_{1} \cdots & C_{p-3} \\
& =-\sum_{j} e_{1} b_{1}^{(n-j)(p-1)-1} \otimes(-1)^{j} \beta \rho^{j}\left(M M L^{p-3}\right) \\
&
\end{aligned}
$$

where $M M L^{p-3}=M_{i-2, i-1} M_{i-3, i-1} L_{i-1}^{p-3}$.

5.5. Lemma. There exists $u \in H^{*}\left(\mathcal{S}_{p^{i}}\right)$ such that $k_{i, i}^{*}(u)=Q_{i-1, i}$.

Proof. The proof is similar to that of 5.4. We let $u^{\prime} \in H^{*}\left(\mathcal{S}_{p^{\prime-1}}\right)$ be such that $k_{i-1, i-1}^{*}\left(u^{\prime}\right)=Q_{i-2, i-1}$ and $u \in H^{*}\left(\mathcal{S}_{p^{\prime}}\right)$ be such that $k_{i-1, i}^{*}(u)=$ $\left(Q_{i-2, i-1}\right)^{\otimes_{p}} \in H^{*}\left(T_{i-1, i}\right)$. Then $k_{i, i}^{*}(u)$ is the $\mathrm{GL}(i, Z / p)$ invariant class containing $(1 \times \Delta)^{*}\left(\mathscr{P}\left(u^{\prime}\right)\right)$. But [8] proved $Q_{i-1, i}$ is the only $\mathrm{GL}(i, Z / p)$ invariant polynomial in this dimension. Thus $k_{i, i}^{*}(u)=c Q_{i-1, i}$, where $c$ is a constant. Note $(1 \times \Delta)^{*}\left(\mathscr{P}\left(u^{\prime}\right)\right)$ contains the term $w_{0} \otimes D_{0}\left(Q_{i-2, i-1}\right)=$ $\left(Q_{i-2, i-1}\right)^{p} \neq 0$. Hence $c \neq 0$.

The naturality of the Steenrod algebra implies image $k_{i, i}^{*}$ contains $\mathcal{Q}(p)\left(M_{i-1, i} M_{i-2, i} L_{i}^{p-3}, Q_{i-1, i}\right)$. By Theorem $\mathrm{E}$ any generator $W_{i}$ is contained in $\mathcal{Q}(p)\left(M_{i-1, i} M_{i-2, i} L_{i}^{p-3}, Q_{i-1, i}\right)$ (see the diagram after Theorem E). This completes the proof of Lemma 5.1.

By 4.5, to complete the proof of Theorem A it suffices to construct a 1-1 correspondence between nonzero monomials in $\mathscr{W}_{i}$ and admissible monomials in $Q(p)$.

5.6. LEMMA. $M_{i-1, i} M_{i-2, i} \cdots M_{1, i} \underline{L}_{i} \neq 0$.

Proof. The term $e_{1} e_{2} \cdots e_{i}\left(b_{1}^{p^{i-1}}\right)^{i-1}\left(b_{2}^{p^{i-2}}\right)^{i-1} \cdots\left(b_{i}\right)^{i-1}$ appears with coefficient 1 in the term-by-term expansion of $M_{i-1, i} M_{i-2, i} \cdots M_{1, i} \underline{L}_{i}$.

The only admissible monomials of length 1 in $\mathcal{Q}(p)_{2 n}$ are $\mathcal{P}^{n-j}\left(u_{2 n}\right)$ and $\beta^{\mathcal{P}^{n-j}}\left(u_{2 n}\right)$ which correspond to $\left(L^{p-1}\right)^{j}$ and $\left(\underline{L}_{1} L_{1}^{p-2}\right)\left(L_{1}^{p-1}\right)^{j-1}$ in $\mho_{i}$. Thus we may assume, by induction, that an $i-1$ length admissible monomial in $\mathcal{Q}(p)_{2 n}$ starting with $\mathcal{P}^{n-j}\left(u_{2 n}\right)$ corresponds to a $j$-fold product monomial in $w_{i-1}(j<n)$. Let $A$ be an admissible monomial in $\mathcal{Q}(p)_{2 n}$.

Case 1. $e_{1}=0$; that is, $A=\beta^{e_{i} \rho s_{i}} \cdots \beta^{e_{2} \rho \rho s_{2}} \rho \rho^{n-j}\left(u_{2 n}\right)$. The dimension of $\mathcal{P}^{n-j}\left(u_{2 n}\right)$ is $2 p(n-j)+2 j$ and hence $s_{2}=p(n-j)+k, 0 \leqslant k \leqslant j$, if $A\left(u_{2 n}\right)$ is nonzero and admissible. Consider 


$$
A^{\prime}=\beta^{e_{i} \odot s_{i}} \cdots \beta^{e_{2} \odot s_{2}}\left(\bar{u}_{2(p(n-j)+j)}\right) \quad \text { where } \bar{u}_{2(p(n-j)+j)}=\mathscr{P}^{n-j}\left(u_{2 n}\right) .
$$

$A^{\prime}$ is an admissible monomial of length $i-1$ and $s_{2}=(p(n-j)+j)-(j-$ $k$ ). Thus $A^{\prime}$ corresponds to a $(j-k)$-fold product monomial in $\mho_{i-1}$, call it $U_{j-k}$. Identify $A$ with $\bar{U}_{j-k}\left(Q_{i-1, i}\right)^{k}$ in $W_{i} . \bar{U}_{j-k}$ comes from $U_{j-k}$ by changing the detecting index from $i-1$ to $i$; i.e., $Q_{m, i-1} \rightarrow Q_{m, i}$.

Case 2. $e_{1}=1$; that is, $A=\beta^{e_{i} \odot s_{i}} \cdots \beta^{e_{2} \rho s_{2}} \beta^{\mathcal{P}^{n-j}}\left(u_{2 n}\right)$. Then consider that part of $A$ until a second Bockstein occurs.

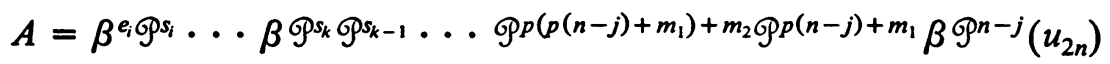

$$
\begin{aligned}
& \text { with } m_{1}>1 \text {. }
\end{aligned}
$$

Further suppose $k<i$. Then

$$
s_{k}=p\left(p\left(p\left(\cdots\left(p(n-j)+m_{1}\right)+m_{2}\right)+\cdots+m_{k-2}\right)+m_{k-1}\right.
$$

and $\mathscr{P}^{s_{k}} \cdots \beta \mathscr{P}^{n-j}\left(u_{2 n}\right)$ has dimension $2 p^{k}(n-j)+2 p^{k-1} m_{1}+2 p^{k-2} m_{2}$ $+\cdots+2 p m_{k-1}+2\left(j-m_{1}-m_{2}-\cdots-m_{k-1}\right)+1$. For $A$ to be admissible and nonzero we must also have $j-m_{1}-m_{2}-\cdots-m_{k-1} \geqslant 0$ and $j-m_{1}-m_{2}-\cdots-m_{k-1}+1 \geqslant 0$. Then

$$
A^{\prime}=\beta^{e_{i}} \mathscr{P} s_{i} \ldots \mathscr{P}^{s_{k+1}}\left(\beta \mathcal{P S}^{s_{k}} \ldots \beta \mathcal{P}^{n-j}\left(u_{2 n}\right)\right)=A^{\prime \prime}\left(\beta \mathcal{P}^{s_{k}} \ldots \beta \beta^{\mathscr{P}^{n-j}}\left(u_{2 n}\right)\right)
$$

and $A^{\prime \prime}$ corresponds to a $j-m_{1}-m_{2}-\cdots-m_{k}+1$ fold product monomial in $\mho_{i-k}$, call it $U_{A^{m}}$. Identify $A$ with the monomial

$$
\bar{U}_{A^{n}}\left(M_{i-k, i} M_{i-1, i} L_{i}^{p-3}\right)\left(Q_{i-k, i}\right)^{m_{k-1}-1}\left(Q_{i-k-1, i}\right)^{m_{k-2}} \cdots\left(Q_{i-2, i}\right)^{m_{2}}\left(Q_{i-1, i}\right)^{m_{1}-1}
$$

where $\bar{U}_{A^{\prime \prime}}$ comes from $U_{A^{\prime \prime}}$ by changing the detecting index from $i-k$ to $i$; i.e., $Q_{m, i-k} \rightarrow Q_{m, i}$. If $k=i$ or no second Bockstein occurs assign to $A$ the monomial

$$
\begin{aligned}
& \left(M_{i-1, i} L_{i} L_{i}^{p-3}\right)\left(L_{i}^{p-3}\right)^{m_{i}}\left(Q_{1, i}\right)^{m_{i-1}} \cdots\left(Q_{i-2, i}\right)^{m_{2}}\left(Q_{i-1, i}\right)^{m_{1}-1} \text { or } \\
& \left(M_{i-1, i} L_{i}^{p-2}\right)\left(L_{i}^{p-3}\right)^{m_{i}}\left(Q_{1, i}\right)^{m_{i-1}} \cdots\left(Q_{i-2, i}\right)^{m_{2}}\left(Q_{i-1, i}\right)^{m_{1}-1} \quad \text { respectively }
\end{aligned}
$$

where $m_{i}=j-m_{1}-m_{2}-\cdots-m_{i-1}$.

Let $U_{A\left(u_{2 n}\right)}$ be the above constructed monomial in $W_{i}$ corresponding to $A\left(u_{2 n}\right)$. It is routine to verify that for $U_{A^{n}}$ in $W_{i-k}$ and $\bar{U}_{A^{n}}$ in $W_{i}$ constructed above we have $\operatorname{dim}\left(U_{A^{\prime \prime}}\right)+2 j\left(p^{i}-p^{i-k}\right)=\operatorname{dim}\left(U_{A\left(u_{2 n}\right)}\right)$. This fact and induction on $i$ show that if $A\left(u_{2 n}\right)$ has dimension $2 n\left(p^{i}\right)-k$ then $U_{A\left(u_{2 n}\right)}$ has dimension $k$. Lemma 5.6 shows $U_{A\left(u_{2 n}\right)} \neq 0$. Hence, by Theorem $4.5,\left(w_{i}\right)_{k}$ must fill out (image $\left.k_{i, i}^{*}\right)_{k}$ for $k \ll n$. This finishes the proof of Theorem A.

VI. Proof of Theorems B, C, D, and F. Consider the following commutative diagram: 


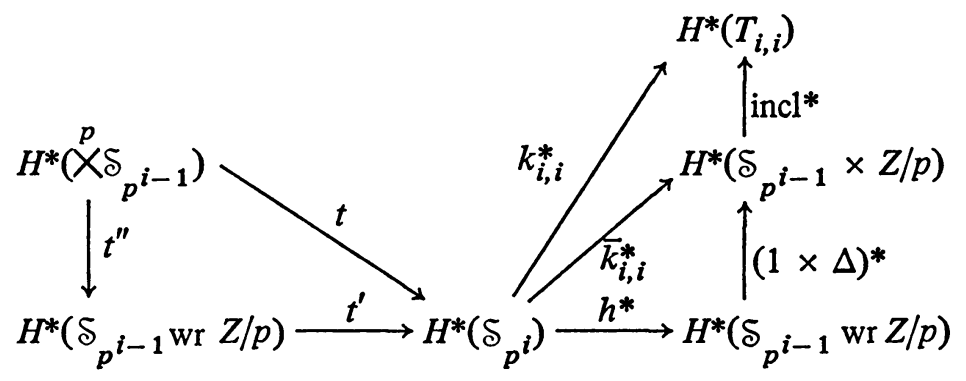

where $h=i\left(\delta_{p^{i-1}}\right.$ wr $\left.Z / p, \delta_{p^{i}}\right)$.

6.1. Proposition. Let $u \in H^{*}\left(\delta_{p^{i}}\right)$. If $k_{i, i}^{*}(u)=0$ then there exists $z \in$ $H^{*}\left(X^{p} \delta_{p^{\prime-1}}\right)$ such that $t(z)=u$.

Proof. By 4.4 and Theorem A, $k_{i, i}^{*}(u)=0$ implies $\bar{k}_{i, i}^{*}(u)=0$. Hence $(1 \times \Delta)^{*} h^{*}(u)=0$ and $h^{*}(u) \in \operatorname{ker}(1 \times \Delta)^{*}$. By 3.1 there exists $z \in$ $H^{*}\left(X^{p}\left(\delta_{p^{\prime-1}}\right)\right)$ such t that $t^{\prime \prime}(z)=h^{*}(u)$. Then $t(z)=t^{\prime} t^{\prime \prime}(u)=t^{\prime}\left(h^{*}(u)\right)=$ $\left[\delta_{p^{\prime}}: \delta_{p^{i-1}} \operatorname{wr} Z / p\right] u=u(\bmod p)$.

Let $u_{s, i-1} \in H^{*}\left(\delta_{p^{i-1}}\right)$, then, by induction, $u_{s, i-1}$ pulls back to a $\delta_{p^{i-1}}$ detecting subgroup $\prod_{l=1}^{q} T_{s_{t}, s_{t}} \rightarrow \delta_{p^{i-1}}$ (recall §II gives these subgroups and their inclusions into $\left.\delta_{p^{i-1}}\right)$. Thus to complete the computation of $H^{*}\left(\delta_{p^{i}}\right)$ it suffices to compute the map $I_{i-1}^{*} t$. First consider the maps $\Phi_{m_{1}, \ldots, m_{n}}=$ $\left(I_{m_{1}, \ldots, m_{n}} \circ\left(\prod_{r=1}^{n}\left(k_{m_{r}, m_{r}}\right)\right)\right)^{*} t_{m_{1}, \ldots, m_{n}}: H^{*}\left(\times_{r=1}^{n} \delta_{p_{r}}\right) \rightarrow H^{*}\left(\mathcal{\delta}_{p}\right) \rightarrow$ $\bigotimes_{r=1}^{n} H^{*}\left(T_{m_{r}, m_{r}}\right)$ for all $\left(m_{1}, \ldots, m_{r}\right)$ such that $\sum_{r=1}^{n} p^{m_{r}}=p^{i}$, with $n \geqslant 2$ and $t_{m_{1}, \ldots, m_{n}}$ the transfer $H^{*}\left(X_{r=1}^{n} \delta_{p^{m_{r}}}\right) \rightarrow H^{*}\left(\delta_{p^{\prime}}\right)$.

6.2. LEMMA. Let $u=u_{1, m_{1}} \otimes \cdots \otimes u_{n, m_{n}} \in H^{*}\left(\times_{r=1}^{n} \delta_{p_{r}}\right)$ and $k_{m_{r}, m_{r}}^{*}\left(u_{r, m_{r}}\right)$ $=v_{r}$. Then

$$
\Phi_{m_{1}, \ldots, m_{n}}(u)=\sum_{\sigma \in \delta_{\left(m_{1}, \ldots, m_{n}\right)}} v_{\sigma(1)} \otimes \cdots \otimes v_{\sigma(n)} .
$$

Proof. As in the proof of 5.2, 2.16 through 2.21 and the following commutative diagram give the proposition:

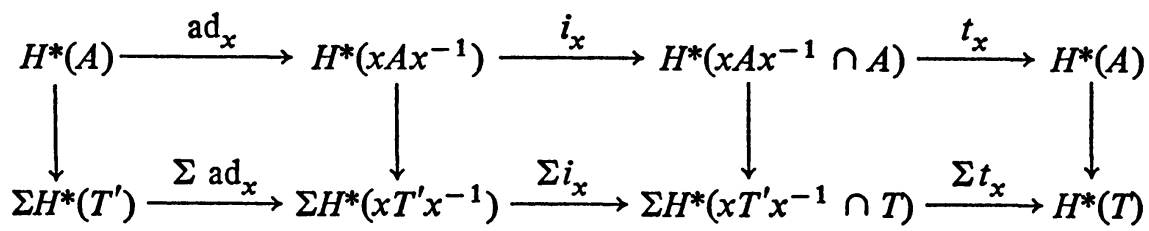

where $A=\times_{r=1}^{n}\left(\mathscr{S}_{p^{m_{r}}}\right), T^{\prime}$ runs through all inclusions of $\times^{m} Z / p$ in $A$ and $T=\times_{r=1}^{n} T_{m_{n, m_{r}}}$.

The only $\delta_{\left(m_{1}, \ldots, m_{n}\right)}$ invariant classes not in image $\Phi_{m_{1}, \ldots, m_{n}}$ are classes $u^{\prime}=u_{1, m_{1}} \otimes \cdots \otimes u_{n, m_{n}}$ containing $\left(u_{r_{0}, m_{10}}\right)^{\otimes p} \in \otimes^{p} H^{*}\left(T_{m_{r_{0}}, m_{r_{0}}}\right)$ as a factor. Recall $u^{\otimes_{p}} \leftarrow^{*} \mathscr{P}(u) \rightarrow^{(1 \times \Delta)^{*}}(1 \times \Delta)^{*}(\mathscr{P}(u))$. Thus $u^{\prime}$ is in the 


$$
\operatorname{image}\left(I_{m_{1}, \ldots, m_{n}} \circ\left(\prod_{r=1}^{n} k_{m_{r}, m_{r}}\right)\right)^{*}: H^{*}\left(\delta_{p^{\prime}}\right) \rightarrow \bigotimes_{r=1}^{n} H^{*}\left(T_{m_{r}, m_{r}}\right) .
$$

Hence we have

6.3. LEMMA. Image $\left(I_{m_{1}, \ldots, m_{n}} \circ\left(\Pi_{r=1}^{n} k_{m_{r}, m_{r}}\right)\right)^{*} \cong \mathcal{S}_{\left(m_{1}, \ldots, m_{n}\right)}$ invariant classes
of $\bigotimes_{r=1}^{n} H^{*}\left(T_{m_{r}, m_{r}}\right)$.

This proves Theorems B and D. A trivial modification of 6.2 and 6.3 proves Theorem F. As 3.1 shows the only multiple image classes are generated by the $\mathscr{P}$ ( )'s, Theorem C follows, up to constants. Using the notation of Theorem C if $x_{m, i-1}=M_{i-2, i-1} M_{i-3, i-1} L_{i-1}^{p-3}$ then 5.4 gives $x_{m, i}=$ $-1\left(M_{i-1, i} M_{i-2, i} L_{i}^{p-3}\right)$. If $x_{m, i-1}=Q_{i-2, i-1}$ then direct computation shows the constant $c$ in 5.5 is 1 hence $x_{m, i}=Q_{i-1, i}$. It is easy to see that application of the Steenrod $p$ th powers or direct computation yield that the constant is +1 for multiple image polynomial generators and -1 for even dimensional multiple image exterior generators.

VII. Proof of Theorem G.

Proof of (1). Let $k_{j, \infty}^{*}(u)=\mathcal{S}\left\langle x_{1}, \ldots, x_{m}, 1, \ldots\right\rangle$. As $j$ is the smallest integer such that $k_{j, \infty}^{*}(u) \neq 0$ it follows that at least one $x_{h}$ contains a factor equal to $L_{j}^{p-1}, \underline{L}_{j} L_{j}^{p-2}, M_{g j} \underline{L}_{j} L_{j}^{p-3}$, or $M_{g j} L_{j}^{p-2}$. If $k_{j, \infty}^{*}(u)$ has at least one representative of the form $\underline{L}_{n, j}(\cdots)$ with $p$ not dividing $n$ then $\beta_{p}\left(k_{j, \infty}^{*}(u)\right)=$ $\sum n L_{n, j}(\cdots)+B \neq 0$ (where $B$ cannot contain terms in the first sum). Similarly if some $x_{h}=M_{g j} L_{j} L_{j}^{p-3} Y$ and no $x_{h^{\prime}}=M_{g, j} L_{j}^{p-2} Y$ then $\beta_{p}\left(k_{j, \infty}^{*}(u)\right)$ $\neq 0$. Suppose every time the term $M_{g j} \underline{L}_{j} L_{j}^{p-3} Y$ appears the term $M_{g j} L_{j}^{p-2} Y$ also appears; then if $k_{j, \infty}^{*}(u) \neq \underline{L}_{n, j}(\cdots) Y$ must be a product of $Q_{h, j}$ 's. It is then easy to construct a class $u^{\prime}$ such that $\beta_{p}\left(u^{\prime}\right)=u$ (just replace one $M_{g j} L_{j}^{p-2} Y$ by $\left.M_{g j} L_{j} L_{j}^{p-3} Y\right)$. If $\beta_{p}(u)=0$ and $M_{g, j} L_{j}^{p-2} Y$ appears a similar construction yields $u^{\prime}$ such that $\beta_{p}\left(u^{\prime}\right)=u$. The only possibility left is $\beta_{p}(u)=0$, and $k_{j, \infty}^{*}(u)=L_{n j}(\cdots)$. Then $\beta_{p}\left(u^{\prime}\right)=u$ where $k_{j, \infty}^{*}\left(u^{\prime}\right)=\underline{L}_{n j}$.

Proof of (2). We need the following

THEOREM [2]. Let $r \geqslant 2$. In homology with the loop sum multiplication if $d^{r-1}(a)=b$ then $d^{r}\left(a^{p}\right)=a^{p-1} b$.

Proof. Theorem 5.4 of [2].

The homology and cohomology Bockstein spectral sequences are Hopf algebra duals and Theorem $\mathrm{F}$ gives the loop sum coalgebra map in cohomology. If $a, b$ in $H_{*}\left(Q\left(S^{0}\right)_{0}\right)$ are dual to $u, v$ respectively then Theorem $\mathrm{F}$ gives $\left\langle u^{\prime}, a^{p}\right\rangle=1$. Now $u^{\prime}$ is not dual to $a^{p}$ on the $E_{1}$ level; in fact $\left(u^{\prime}\right)^{*}=a^{p}+$ $\sum a_{i}$. It is easy to see however that the $a_{i}$ are all dual to classes $u^{\prime \prime}$ where $k_{j, \infty}^{*}\left(u^{\prime \prime}\right)=\delta\left\langle x_{1}, \ldots, x_{t}, 1, \ldots\right\rangle$ with $t<p m$. 
Many times it is easy to see that the $a_{i}$ classes do not live to $E_{r}$. Such is the case with Corollary 1 as induction on $r$ and the fact that $\left\{L_{p^{m}, j}(x\right.$ : $1, \ldots)\}_{m=1}^{r-1}$ generate the subalgebra $\left\{L_{n, j}(x: 1, \ldots)\right\}$ (where $n=1, \ldots$, $\left.p^{r}-1\right)$ prove the corollary.

Proof of Corollary 2. The reduction homomorphism $j_{r}: H^{*}\left(, Z / p^{\prime}\right) \rightarrow$ $E_{r}$ is onto and if $k_{i, i}^{*}(u)=Q_{j, i}$ then $k_{j, i}^{*}(u)=R_{i}^{*}\left(L_{p^{j}, j}(1: 1, \ldots)\right)$.

Appendix. We give a proof that the quotient determinants, $Q_{j, i} \in \mathcal{W}_{i}$ a're integral mod $p . L_{i}$ has an explicit factorization first discovered by $\mathrm{E}$. $\mathrm{H}$. Moore in 1896

LEMMA [19]. $L_{i}=\Pi_{\left(m_{1}, \ldots, m_{i}\right)}\left(m_{1} b_{1}+\cdots+m_{i} b_{i}\right)$ where $\left(m_{1}, \ldots, m_{i}\right)$ runs over all elements of $T_{i, i}$ with first nonzero coefficient equal to one.

Proof. (Compare with [8, p. 76].) $L_{i}$ is invariant under the special linear group $\operatorname{SL}(i, Z / p)$ which acts transitively on the nonzero elements of $T_{i, i}$. Since $b_{1}$ is a factor of $L_{i}$ it follows that $\alpha\left(b_{1}\right)=m_{1} b_{1}+\cdots+m_{i} b_{i}$ is a factor as well. Hence the product above divides $L_{i}$ (the factors are all relatively prime). But both sides have the same degree, hence they differ only up a constant factor. But the diagonal term $b_{1}^{p^{i-1}} b_{2}^{p^{i-2}} \cdots b_{i}$ occurs in both sides only once and each time with coefficient 1 .

More generally $b_{1}$ is a factor of the numerator of $Q_{j, i}$ for every $j$, so $L_{i}$ is also a factor of the numerator of $Q_{j, i}$ by the above argument. This gives:

LEMMA. $Q_{j, i}$ is a nontrivial polynomial invariant under $\mathrm{GL}(i, Z / p)$.

\section{REFERENCES}

1. S. Araki and T. Kudo, Topology of $H_{n}$-spaces and $H$-squaring operations, Mem. Fac. Sci. Kyusyu Univ. Ser. A 10 (1956), 85-120.

2. W. Browder, Homotopy commutative H-spaces, Ann. of Math. (2) 75 (1962), 283-311. MR 27 \#765.

3. G. Brumfiel, I. Madsen, and R. J. Milgram, PL-characteristic classes and cobordism, Ann. of Math. (2) 97 (1973), 82-159.

4. H. Cárdenas, El algebra de cohomologia del grupo simétrico de grado $p^{2}$, Bol. Soc. Mat. Mexicana 10 (1965), 1-30.

5. H. Cartan and S. Eilenberg, Homological algebra, Princeton Univ. Press, Princeton, N. J., 1956. MR 17, 1040.

6. A. Cauchy, Exercises d'analyse et de physique mathématique. III, Paris, 1844.

7. L. E. Dickson, The Madison Colloquium Lectures on Mathematics, Amer. Math. Soc. Colloq. Publ., vol. 4, Amer. Math. Soc., Providence, R. I., 1913, pp. 33-40.

8. , A fundamental system of invariants of the general modular linear group with a solution of the form problem, Trans. Amer. Math. Soc. 12 (1911), 75-98.

9. A. Dold, Homology of symmetric products and other functors of complexes, Ann. of Math. (2) 68 (1958), 54-80. MR 20 \#3537. 
10. E. Dyer and R. Lashof, Homology of iterated loop-spaces, Amer. J. Math. 84 (1962), 35-88. MR 25 \#4523.

11. S. Eilenberg and S. Mac Lane, On the group $H(\pi, n) I$, Ann. of Math. (2) 58 (1953), 55-106. MR 15, 54.

12. H. Hopf, Über die Bettischen Gruppen, die zu einer beliebigen Gruppe gehören, Comment. Math. Helv. 17 (1945), 39-79. MR 6, 279.

13. I. Madsen and R. J. Milgram, The universal smooth surgery class, Comment. Math. Helv. 50 (1975), 281-310. MR 52\#4285.

14. , Higher torsion in SG and BSG, Math. Z. 143 (1975), 55-80. MR 51 \# 11503.

15. J. P. May, Homology operations on infinite loop spaces, Algebraic topology (Proc. Sympos. Pure Math., Vol. 22, Univ. Wisconsin, Madison, Wis., 1970), Amer. Math. Soc., Providence, R. I., 1971, pp. 171-185. MR 47 \#7740.

16. R. J. Milgram, The mod 2 spherical characteristic classes, Ann. of Math. (2) 92 (1970), 238-261. MR 41 \# 7705.

17. __ The homology of symmetric products, Trans. Amer. Math. Soc. 138 (1969), 251-265. MR 39 \#3483.

18. __ Surgery with coefficients, Ann. of Math. (2) 100 (1974), 194-248. MR 50 \# 14801.

19. E. H. Moore, $A$ two-fold generalization of Fermat's theorem, Bull. Amer. Math. Soc. 2 (1896), 189-199.

20. M. Morse, The calculus of variations in the large, Amer. Math. Soc. Colloq. Publ., vol. 18, Amer. Math. Soc., Providence, R. I., 1934.

21. M. Nakaoka, Decomposition theorem for homology groups of symmetric groups, Ann. of Math. (2) 71 (1960), 16-42. MR 22 \#2989.

22. , Homology of the infinite symmetric group, Ann. of Math. (2) 73 (1961), 229-257. MR 24 \#A1721.

23. G. Nishida, Cohomology operations in iterated loop spaces, Proc. of the Japan Acad. 44 (1968), 104-109. MR 39 \#2156.

24. S. Priddy, On $\Omega^{\infty} S^{\infty}$ and the infinite symmetric group, Algebraic topology (Proc. Sympos. Pure Math., Vol. 22, Univ. Wisconsin, Madison, Wis., 1970), Amer. Math. Soc., Providence, R. I., 1971, pp. 217-220. MR 50 \# 11226.

25. D. Quillen, Unpublished.

26. , On the cohomology and $K$-theory of the general linear groups over a finite field, Ann. of Math. (2) 96 (1972), 552-586. MR 47 \#3565.

27. __ The Adams conjecture, Topology 10 (1971), 67-80.

28. P. A. Smith and M. Richardson, Periodic transformations of complexes, Ann. of Math. (2) 39 (1938), 611-633.

29. N. E. Steenrod, Products of cocycles and extensions of mappings, Ann. of Math. (2) 48 (1947), 290-320. MR 9, 154.

30. Homology groups of symmetric groups and reduced power operations, Proc. Nat. Acad. Sci. U.S.A. 39 (1953), 213-217. MR 14, 1005.

31. N. E. Steenrod and D. B. A. Epstein, Cohomology operations, Ann. of Math. Studies, No. 50, Princeton Univ. Press, Princeton, N. J., 1962. MR 26 \#3056.

32. J. Tornehäve, Developing the Quillen map, Thesis, M. I. T., 1971.

33. A. Tsuchiya, Characteristic classes for spherical fiber spaces, Nagoya Math. J. 43 (1971), 1-39. MR 45 \#7736.

34. Characteristic classes for PL micro bundles, Nagoya Math. 43 (1971), 169-198. MR 47, 2614.

35. B. Cooper, Cohomology of symmetric groups, Augsberg College, 1975 (mimeo).

36. H. Mui, Modular invariant theory and cohomology algebras of symmetric groups, J. Fac. Sci. Univ. Tokyo 22 (1975), 319-369.

37. F. Cohen, T. Lada and J. P. May, The homology of iterated loop spaces, Lecture Notes in Math., vol. 533, Springer-Verlag, Berlin and New York, 1976. 
38. H. Ligaard, On the Adams spectral sequence for $\Pi_{x}(M S T O P)$ and infinite loop maps from $S F$ to $\mathrm{BO}_{\otimes}$ at the prime 2, Thesis, University of Chicago, 1977.

39. B. Mann and R. J. Milgram, On the Chern classes of the regular representations of some finite groups, Stanford Univ., 1975 (mimeo).

Department of Mathematics, Stanford University, Stanford, California 94305

Current address: Department of Mathematics, Harvard University, Cambridge, Massachusetts 02138 